\title{
Characterization and error analysis of an $N \times N$ unfolding procedure applied to filtered, photoelectric x-ray detector arrays. I. Formulation and testing
}

\author{
D. L. Fehl, G. A. Chandler, W. A. Stygar, R. E. Olson, C. L. Ruiz, J. J. Hohlfelder, L. P. Mix, and F. Biggs \\ Sandia National Laboratories, Albuquerque, New Mexico 87185, USA
}

M. Berninger, P. O. Frederickson, and R. Frederickson

National Security Technologies, LLC, Los Alamos, New Mexico 87544, USA

(Received 18 September 2009; published 30 December 2010)

\begin{abstract}
An algorithm for spectral reconstructions (unfolds) and spectrally integrated flux estimates from data obtained by a five-channel, filtered x-ray-detector array (XRD) is described in detail and characterized. This diagnostic is a broad-channel spectrometer, used primarily to measure time-dependent soft $\mathrm{x}$-ray flux emitted by $z$-pinch plasmas at the $Z$ pulsed-power accelerator (Sandia National Laboratories, Albuquerque, New Mexico, USA), and serves as both a plasma probe and a gauge of accelerator performance. The unfold method, suitable for online analysis, arises naturally from general assumptions about the x-ray source and spectral properties of the channel responses; a priori constraints control the illposed nature of the inversion. The unfolded spectrum is not assumed to be Planckian. This study is divided into two consecutive papers. This paper considers three major issues: (a) Formulation of the unfold method.-The mathematical background, assumptions, and procedures leading to the algorithm are described: the spectral reconstruction $S_{\text {unfold }}(E, t)$-five histogram x-ray bins $j$ over the x-ray interval, $137 \leq E \leq 2300 \mathrm{eV}$ at each time step $t$-depends on the shape and overlap of the calibrated channel responses and on the maximum electrical power delivered to the plasma. The $\mathrm{x}$-ray flux $\mathcal{F}_{\text {unfold }}$ is estimated as $\int S_{\text {unfold }}(E, t) d E$. (b) Validation with simulations.-Tests of the unfold algorithm with known static and time-varying spectra are described. These spectra included—but were not limited to-Planckian spectra $S_{b b}(E, T)(25 \leq T \leq 250 \mathrm{eV})$, from which noise-free channel data were simulated and unfolded. For Planckian simulations with $125 \leq T \leq 250 \mathrm{eV}$ and typical responses, the binwise unfold values $S_{j}$ and the corresponding binwise averages $\left\langle S_{b b}\right\rangle_{j}$ agreed to $\sim 20 \%$, except where $S_{b b} \ll \max \left\{S_{b b}\right\}$. Occasionally, unfold values $S_{j} \lesssim 0$ (artifacts) were encountered. The algorithm recovered $\gtrsim 90 \%$ of the x-ray flux over the wider range, $75 \leq T \leq 250 \mathrm{eV}$. For lower $T$, the test and unfolded spectra increasingly diverged as larger fractions of $S_{b b}(E, T)$ fell below the detection threshold $(\sim 137 \mathrm{eV}$ ) of the diagnostic. (c) Comparison with other analyses and diagnostics. - The results of the histogram algorithm are compared with other analyses, including a test with data acquired by the DANTE filtered-XRD array at the NOVA laser facility. Overall, the histogram algorithm is found to be most useful for x-ray flux estimates, as opposed to spectral details. The following companion paper [D. L. Fehl et al., Phys. Rev. ST Accel. Beams 13, 120403 (2010)] considers (a) uncertainties in $S_{\text {unfold }}$ and $\mathcal{F}_{\text {unfold }}$ induced by both data noise and calibrational errors in the response functions; and (b) generalization of the algorithm to arbitrary spectra. These techniques apply to other diagnostics with analogous channel responses and supported by unfold algorithms of invertible matrix form.
\end{abstract}

DOI: 10.1103/PhysRevSTAB.13.120402

PACS numbers: 52.70.La, 52.59.Qy, 52.58.Lq, 52.50.-b

\section{INTRODUCTION}

High-temperature plasma, created in the laboratory, has been a topic of keen interest in physics for over 50 years [1-4]. Particularly tantalizing are hot, optically dense plasmas, in which radiation transport may be studied close at hand and which may approach stellar conditions [5-9]. Such plasmas have been produced on short time scales ( $\lesssim 10 \mathrm{~ns}$ ) by intense, focused laser beams [10-12], particle beams $[13,14]$, and most recently by $z$ pinches of tungsten-wire arrays [15-18]. Besides their intrinsic interest to physics, these plasmas also emit copious amounts of soft $\mathrm{x}$ rays $(\sim 50 \mathrm{eV}-3 \mathrm{keV})$, finding application as sources for opacity measurements [9], drivers for inertial confinement fusion (ICF) [19-23], vulnerability testing of materials [24,25], and equation-of-state research [26,27].

Obtaining information about the time-dependent $\mathrm{x}$-ray spectrum [28] emitted by such sources is an important diagnostic goal. The spectral shape gives clues to particle interactions and to processes of $\mathrm{x}$-ray production and propagation in the plasma. The spectrally integrated $\mathrm{x}$-ray flux [28] assesses the radiated x-ray power from the plasma as well as the average energy of particles within it. A measurement of $\mathrm{x}$-ray flux also permits one to ascribe a radiation (brightness) temperature to plasmas and hohlraums. In addition, some operational behavior of the 
plasma generator may be assessed via $\mathrm{x}$-ray emission measurements.

Time-resolved, absolute, soft $x$-ray flux diagnostics form the context of this article. Such measurements are not trivial to make, especially in the severe environment that accompanies pulsed-power-generated z-pinch plasmas, where the pulse widths (FWHM) are short $(\sim 6 \mathrm{~ns})$ $[15,16]$, and the $\mathrm{x}$-ray irradiance is intense [15,29] $\left(\sim 10^{6} \mathrm{~W} \mathrm{~cm}^{-2}\right.$ at $24 \mathrm{~m}$ from the source [30]). Under these conditions, the detectors run in current mode. Soft $\mathrm{x}$-ray spectra pose additional problems for flux measurements because photon detection in the sub-to-few $\mathrm{keV}$ range occurs via the photoelectric effect, which varies strongly and discontinuously with $\mathrm{x}$-ray energy. Hence, unless a single, soft-x-ray detector is made totally absorbing or its sensitivity is somehow flattened, it will not isolate spectrally integrated flux from spectral shape. Some singledetector-based diagnostics have, in fact, addressed flux measurements in this way [31-34].

Encoding [35] is another method of addressing detector sensitivity variations within flux measurements. In this technique, the incident $\mathrm{x}$-ray spectrum is spread out over an array of $N$ detectors so that each detector samples $\mathrm{x}$ rays over a limited, but prescribed, spectral interval. The coding process associates partial flux in a spectral interval with one or more detectors, called channels. In principle, if the incident spectrum does not vary significantly within these $\mathrm{x}$-ray intervals, variations in detector sensitivity become less troublesome than for a single, bare detector used over a wider energy range. If the mapping can be inverted, the integrated x-ray flux may be estimated from the channel data. Wavelength dispersive techniques [25,36-39] are widely used examples of this method.

The particular subject of this article is a variant of the $N$-detector encoding method for measuring $x$-ray flux. In this technique, each detector in an array views the $\mathrm{x}$-ray source through its own separate filter. These filterdetector pairs $(i=1, \ldots, N)$ view the source at nearly the same solid angle. The attenuation of the filter and the sensitivity of detector then define an overall spectral response function $R_{i}(E)$ for each such channel, which can be designed to isolate a specific portion(s) of an incident spectrum and can be absolutely calibrated experimentally-though, by spectroscopic standards, the resolution obtained is poor $(E / \Delta E \approx 1)$, and the filtered-detector responses often overlap. Figure 1 illustrates five response functions, used in this article, that define several superposed regions of x-ray sensitivity (or, so-called, "spectral cuts") between $100 \mathrm{eV}$ and $5 \mathrm{keV}$. Similarly shaped responses have been reported for a variety of detectors combined as simple arrays with upstream filters and in differing spectral ranges [40-45]. Improvements to this basic design for soft $\mathrm{x}$ rays have been achieved by inserting additional x-ray components, upstream of the detectors [46-48].

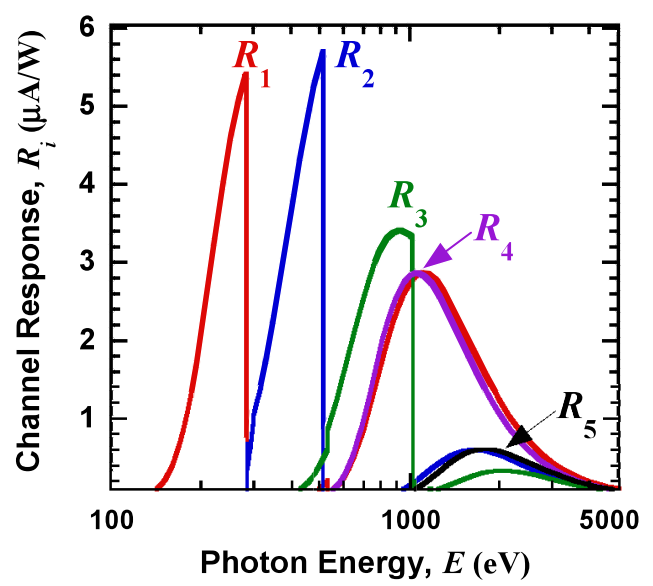

FIG. 1. A representative set of response functions, $R_{1}(E), \ldots, R_{5}(E)$, as a function of incident x-ray energy $E$, for the filtered-XRD $Z$ diagnostic. The sharp features correspond to characteristic X-ray absorption edges in the upstream filters and the XRD photocathodes. At low X-ray energies the responses together form three energy-selective, sensitivity bands (spectral "cuts") which, however, overlap at higher x-ray energies and decrease by roughly another order of magnitude as $E \rightarrow \sim 30 \mathrm{keV}$ [Ref. [64]].

Yet, encoding a spectrum is only half the problem. Decoding channel data for spectral information is also challenging. Why this should be so may also be inferred from Fig. 1. (a) The responses $R_{i}(E)$ available from x-ray filtration only coarsely divide the spectral range and are relatively insensitive to spectral details on a finer scale; hence, the data coding is ambiguous for spectra that may differ in relatively narrow details. (b) The $R_{i}(E)$ 's are energy dependent and still spectrally bias the channel data-though to a lesser extent than a bare detector. (c) Overlap of the responses raises the issue that some of the channel data may be redundant, also degrading spectral discrimination. (d) Added to these issues are channel-wise perturbations due to signal noise and calibrational uncertainties, neither of which need directly relate to the $\mathrm{x}$ rays under study. All these effects complicate, even frustrate, attempted reconstructions (or unfolds) of a spectrum incident on such filtered-detector-array diagnostics.

The specific focus of this article is an absolutely calibrated, filtered, photoelectric-x-ray-detector (XRD) array, routinely fielded at the $Z$ accelerator [15-18] (Sandia National Laboratories, Albuquerque, NM, USA). The principal task of this instrument, hereafter called the $Z$ diagnostic, is to estimate spectrally integrated flux of continuum $\mathrm{x}$ rays emitted by $z$-pinch plasmas and hohlraums. The information obtained is used to study the plasmas produced by the $Z$ accelerator and to optimize X-ray production [17].

This article describes and characterizes the unfold algorithm for the $Z$ diagnostic. Although many unfold techniques $[49,50]$ may potentially be harnessed for inverting channel data from this diagnostic, we show here that its 
response functions together with reasonable assumptions about the X-ray source permit a straightforward unfold algorithm with sufficient detail [51,52] to make the assigned flux estimate-especially at peak emitted x-ray power. The algorithm is, in fact, semiclassical (an $N \times N$, directly inverted, product-moment method with a priori constraints, histogram basis functions, and collocated data $[49,53,54])$ and can be implemented on a real-time basis. Previous publications [14,29,55-57] have sketched this algorithm, but no justification or detailed testing of the
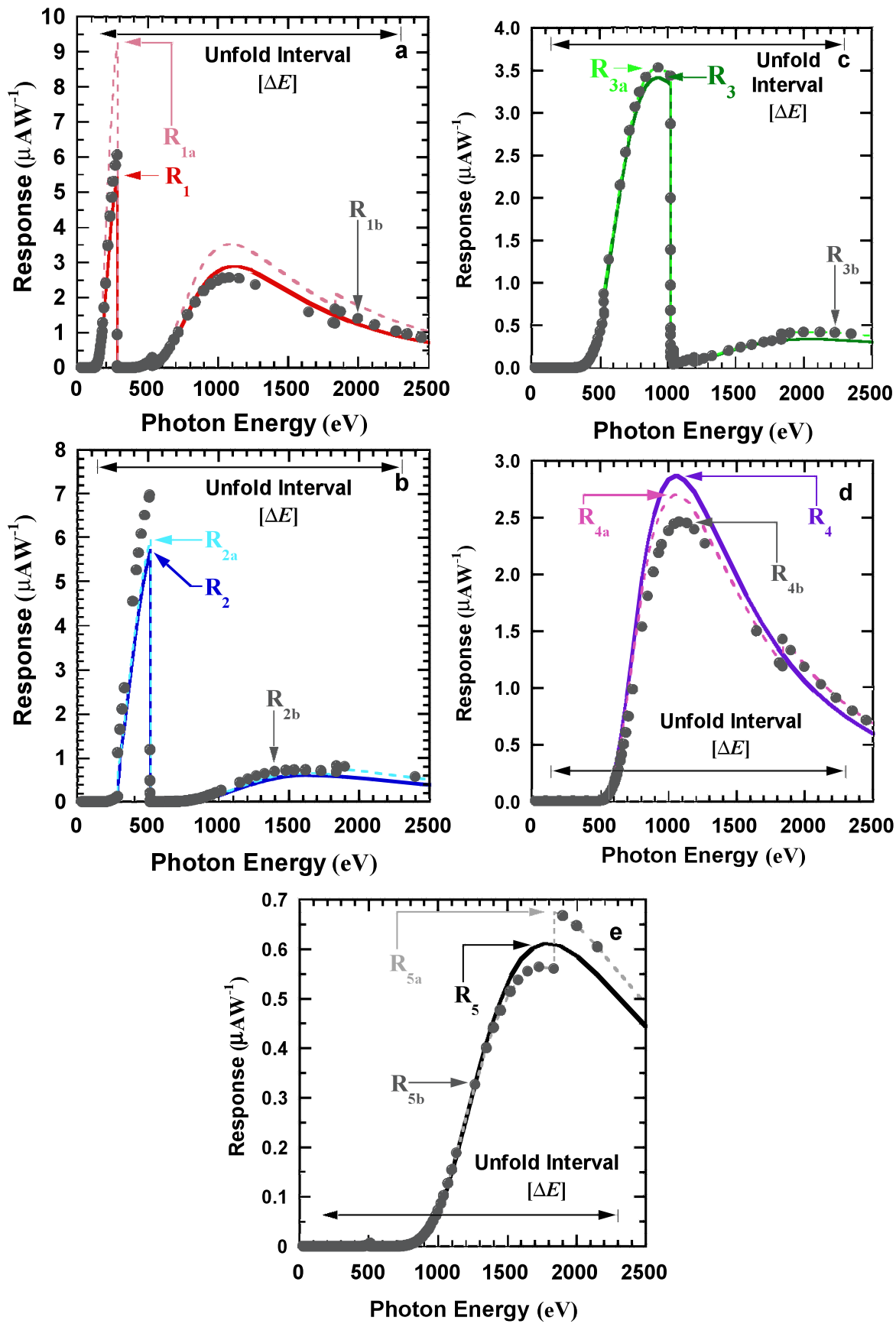

FIG. 2. Stability and reproducibility of the channel responses. The three curves in each frame are curve fits to calibrational x-ray data (not shown). Each frame includes (a) the reference calibration $R_{i}(E)$ for the $Z$ diagnostic (solid curve), (b) a subsequent calibration $R_{i a}(E)$ after several months' service, and (c) a calibration $R_{i b}(E)$ with different physical components (gray dots). Differences between $R_{i}$ and $R_{i a}$ indicate a drift in the diagnostic between calibrations. The jump in the sensitivity of $R_{i a}$ at $1839 \mathrm{eV}$, not visible in $R_{i}$, is due to $\mathrm{Si}$ adsorbed onto the XRD's from the vacuum system. A comparison of $R_{i}$ and $R_{i b}$ shows how closely $R_{1}, \ldots, R_{5}$ can be reproduced with different XRD's and filters. The unfold interval, $[\Delta E]=[137,2300] \mathrm{eV}$, is indicated in each frame. 
method has been reported. Thus, the purpose of this article is (a) to give a complete account of the algorithm (formulation, simulations, experimental comparisons, and error analysis), (b) to show how and why this particular algorithm responds to arbitrary spectral functions, and (c) to point out major pitfalls associated with this and other spectral inversions for similar diagnostics. Since this algorithm is not a panacea, alternative methods are noted. The intended audience comprises those who field filtereddetector diagnostics, those who use the results of such diagnostics in pulsed-beam plasma experiments, and those considering the use of these techniques. (A separate procedure for estimating $\mathrm{x}$-ray flux from such arrays without unfolding has been reported by Fehl et al. [29].)

This article is divided into two companion papers (denoted as parts 1 and 2), of which this first part considers the formulation and testing of the unfold algorithm. Here, Sec. II presents a detection model for the $Z$ diagnostic (in terms of its response functions), describes the expected spectral properties of $\mathrm{x}$ rays emitted by $z$-pinch plasmas at the $Z$ accelerator, and identifies potential difficulties in reconstructing such spectra from $Z$-diagnostic data. Section III derives the $Z$ unfold algorithm. Major emphasis is placed on evaluating the spectral behavior (domain, condition, and resolution) of the channel-wise responses by applying numerical tools that, we believe, are largely untapped by the pulsed-beam plasma community. These appraisals, together with the a priori spectral information and the goal of estimating x-ray flux, lead in first order to an exactly determined reconstruction in matrix form. (Alternative choices are noted.) Section IV evaluates intrinsic distorting effects of the algorithm for a range of prescribed spectra by simulating $Z$-diagnostic data and then unfolding these data. Both Planckian and nonPlanckian sources are considered over a range of spectra not previously published for such diagnostics. Section V compares the results of the $Z$ algorithm to other unfold and flux-estimation techniques with both simulated [29] and experimental data [58]; included are previously unpublished unfolds of data acquired by the DANTE filteredXRD diagnostic [46,47] at the NOVA Laser Facility $[59,60]$. Concluding remarks and a summary follow these major sections.

Part 2 of this article examines broader issues for the $Z$-unfold algorithm and incorporates analysis not previously reported for the $Z$ diagnostic nor, we believe, for similar filtered-detector arrays applied to pulsed-beam plasma $x$-ray sources. Following a summary of the defining unfold equations in the Introduction, Sec. II examines error propagation and the stability of the unfold in the face of systematic and random perturbations, due to signal noise and calibrational uncertainties. To determine how the $Z$ diagnostic and its unfold method responds to arbitrary spectral sources, Sec. III generalizes the algorithm by eliminating explicit reference to simulated channel data, a step which leads to spectral sensitivity functions $[35,50,61]$ (called here, passband functions) for each parameter of $S_{\text {unfold }}$ and the flux estimate. These tools quantitatively explain properties of the algorithm noted in part 1 and its limitations. A summary and concluding remarks follow in Sec. IV.

Both parts of this article include appendices and informational end notes. References to part 2 of this article are prefixed with "Pt. 2:" (e.g., "cf. Pt. 2: Fig. 2"); references to this part contain no prefix (e.g., "cf. Fig. 2").

\section{MATHEMATICAL BACKGROUND AND ASSUMPTIONS}

(Mathematical notation and definitions pertaining to this article appear in Pt. 2: Table I.)

\section{A. General diagnostic model}

We begin with a formal model for encoding a timevarying x-ray spectrum $S(E, t)$ into experimental channel data $D_{i}(t)$ for an $N$-channel, filtered-detector array. Under controlled conditions, $D_{i}(t)$ can be written as

$$
\begin{aligned}
D_{i}(t) & =\int_{0}^{E_{\mathrm{MAX}}} R_{i}(E) S(E, t) d E+\varepsilon_{i}(t) \\
& =d_{i}(t)+\varepsilon_{i}(t) \quad(i=1, \ldots N),
\end{aligned}
$$

where $E$ is the x-ray energy. The variable $t$ denotes time as an index for corresponding spectra and data. For computational purposes, it is convenient to define $D_{i}(t)\left[\mathrm{A} \mathrm{sr}^{-1}\right]$ as the recorded signal voltage $V_{i}[\mathrm{~V}]$ in the $i$ th XRD channel, divided by the characteristic impedance $z_{0}[\Omega]$ of the recording system and by the solid angle, with a correction for off-normal observation (Appendix A). In the following, $D_{i}(t)$ is sometimes treated as the $i$ th component of an $N$-dimensional, experimental data vector $\mathbf{D}(t)$.

Two contributions [62,63] to the experimental data $D_{i}(t)$ are accounted for in Eq. (1). The first, denoted $d_{i}(t)=\int R_{i}(E) S(E, t) d E$, represents noise-free signal from filtered x rays. Each response function $R_{i}(E)$ quantifies the detector current per unit $\mathrm{x}$-ray power $\left(\mathrm{A} \mathrm{W}^{-1}\right)$ and is known from experimental calibrations (e.g., Fig. 1). The spectrum $S(E, t)$ is the differential energy flux (typically, $\mathrm{W} \mathrm{sr}{ }^{-1} \mathrm{eV}^{-1}$ ), viewed normal to the source. (Alternate units, $\mathrm{W} \mathrm{sr}{ }^{-1} \mathrm{eV}^{-1} \mathrm{~cm}^{-2}$, may be used for known source area.) Physically admissible functions, $R_{i}(E)$ and $S(E, t)$, are non-negative (cf. Secs. II B and II E for further restrictions.) The integral in Eq. (1) is taken over x-ray energies up to some cutoff $E_{\mathrm{MAX}}$, above which the responses are deemed insensitive to $\mathrm{x}$ rays or the differential spectrum disregarded. On the basis of Fig. 1, one might choose, e.g., $E_{\mathrm{MAX}} \geq 5 \mathrm{keV}[64,65]$. However, approximating $S(E, t)$ over a smaller interval may prove adequate for many experiments (cf. Sec. III A). [The same notation, $d_{i}(t)$, applies if $S(E, t)$ is replaced by less restrictive spectral functions, $f(E, t)$.] 
The second contributor to $D_{i}(t)$ in Eq. (1) is $\varepsilon_{i}(t)$, which represents channel-dependent processes that perturb the underlying x-ray signal $d_{i}(t)$ and includes geometrical errors, signal noise, and calibrational uncertainties. $\varepsilon_{i}$, unlike $d_{i}$, may assume negative values. Any unfolding algorithm of Eq. (1) must, therefore, cope with perturbed channel data $D_{i}(t)$, since the noise-free x-ray data $d_{i}(t)$ are not known (cf. Pt. 2: Sec. II).

Overall, Eq. (1) is linear in both contributions. Thus, e.g., if $S_{1}$ and $S_{2}$ are simultaneously imposed spectra and $\lambda$ is a scalar, then $d_{i}\left[S_{1}+\lambda S_{2}\right]=d_{i}\left[S_{1}\right]+\lambda d_{i}\left[S_{2}\right]$ obtains. This condition places x-ray flux limitations on the $Z$ diagnostic [62] (cf. Appendix B).

\section{B. Known properties of $\boldsymbol{R}_{i}$, expectations for $S$, and typical data $D_{i}$ in $z$-pinch experiments}

Chandler [56,66] has described the construction, calibration, and fielding of the $Z$ diagnostic, which is based on earlier designs $[67,68]$. This x-ray probe comprises an array of five, independently filtered, carbon XRD's. Such detectors are generally fast and have a large, linear dynamic range. The designed spectral range of the $Z$ diagnostic is $\sim 100 \mathrm{eV}-2 \mathrm{keV}$. Similar, but more sophisticated, diagnostics have been reported, specifically, by Kornblum [46] and Kauffman [47] ("DANTE", 15 channels between $\sim 100-3 \mathrm{keV}$ ) and by Bourgade [48] ("DMX", 18 channels between $\sim 50 \mathrm{eV}-20 \mathrm{keV}$ ).

The absolute calibration of the $Z$ diagnostic depends on characterizations of the XRD's and upstream filters. Each response function $R_{i}(E)$ is modeled as the product of two separate and independent measurements:

$$
R_{i}(E) \equiv \chi_{i}(E) \Theta_{i}(E)
$$

Here, $\chi_{i}(E)$ is the spectral current sensitivity of the $i$ th XRD to incident X-ray power, and $\Theta_{i}(E)$ is the net spectral transmission of the corresponding upstream filter-sometimes composite. Both functions are based on least-squares fits to calibrational data (cf. Appendix B).

Figures 2(a)-2(e) illustrate the stability and reproducibility of channel responses nominally used for the $Z$ diagnostic. Three calibrational fit curves are shown for each channel. The solid lines, labeled $R_{1}(E), \ldots, R_{5}(E)$, represent $\chi_{i}(E) \Theta_{i}(E)$ for a particular set of new filters and carbon XRDs. These curves (on a linear scale) are the same as shown in Fig. 1 and are used here and elsewhere [29,55] as reference responses. The dashed curves, denoted $R_{1 a}, \ldots, R_{5 a}$, were obtained for the same XRD's after extended service at the $Z$ accelerator in vacuum environments of $\sim 10 \mu$ Torr. One notes a calibrational drift, $R_{i}(E) \rightarrow R_{i a}(E)$, especially in channels 1 and 5 . The gray dotted curves, $R_{1 b}, \ldots, R_{5 b}$, show how closely the reference responses can be matched with replacement parts. For unfolding purposes, it is more important to have accurate channel calibrations than to duplicate the specific functions, $R_{1}(E), \ldots, R_{5}(E)$, exactly [cf. Fig. 10(a) and Pt. 2: Sec. II B].

The five-channel filtered-XRD array is one of several diagnostics applied to $z$-pinch plasmas at the $Z$ accelerator [15-18,25,30,32-34,38,39]. This pulsed-power generator delivers up to $20 \times 10^{6} \mathrm{~A}$ and $3 \times 10^{6} \mathrm{~J}$ to a load composed of hundreds of coaxial wires (typically tungsten, total mass $\sim 1 \mathrm{mg}$ ) $[9,15-17,23,69,70]$. Over a time scale of $\sim 100 \mathrm{~ns}$, the wires vaporize, implode, and stagnate on axis, creating a roughly cylindrical, x-ray-emitting plasma $(\sim 2 \mathrm{~mm}$ diameter, length 1-2 cm, and lifetime of 5.5-10 ns, FWHM). The current-generated B-field confines, compresses, and heats the $z$ pinch. X-ray framing camera pictures depict a spatially complex and time-dependent brightness pattern [15,70,71]. Haines [71] has reviewed progress in understanding the dynamics of such plasmas.

The spectrum $S(E, t)$ of photons emitted by a $z$-pinch plasma depends on the atomic energy levels and density of the ions, their degree of ionization, and the materials surrounding the plasma [6]. The pinch parameters above suggest that at stagnation, tungsten $z$-pinch plasmas may qualify as both hot (ion and electron temperatures, $T_{i}, T_{e}>$ $100 \mathrm{eV})$ and dense $\left(>10^{18}\right.$ ions $\left./ \mathrm{cm}^{3}\right)$ where local-thermodynamic-equilibrium conditions prevail. Hence, one expects $S(E, t)$ versus $E$ to be largely continuous, but generally punctuated by both emission and absorption features $[5-7,25,72]$. In the absence of thermal gradients within the tungsten plasma and its environment, $S(E, t)$ is often approximated by a time-varying, blackbody (Planckian) spectrum [9], with possibly a non-Planckian high-energy exponential tail [25,37]. An upper-bound brightness temperature [37] for a $z$ pinch at the $Z$ accelerator may then be estimated by modeling the plasma as a

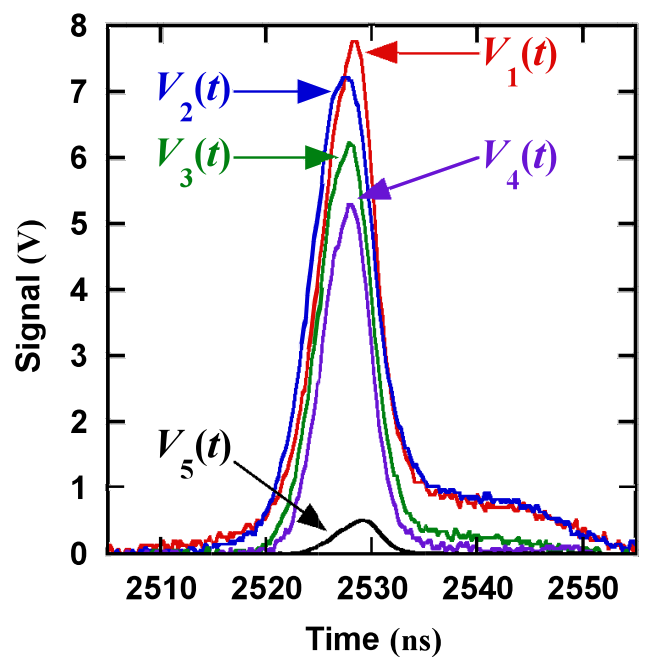

FIG. 3. XRD voltage signals $V_{i}(t)$ for $Z$-shot 165 $\left(R_{1 b}, \ldots, R_{5 b}\right.$, Fig. 2). In this experiment the signal-to-dataconversion factor, $\kappa=D_{i}(t) / V_{i}(t)$, was independent of channel (cf. Appendix A). The signals do not necessarily peak together. 
cylindrically symmetric, blackbody radiator $\left(1.26 \mathrm{~cm}^{2}\right.$ of surface area, as above) in equilibrium with the average incoming electrical power $\left(550 \times 10^{12} \mathrm{~W}=\right.$ $3 \mathrm{MJ} / 5.5 \mathrm{~ns})$. This analysis yields a blackbody temperature of $\sim 250 \mathrm{eV}$, where $\sim 95 \%$ of the total radiated $\mathrm{x}$-ray power is delivered in photons of energy $E \lesssim 1 \mathrm{keV}$. Based on several $x$-ray diagnostics, the peak measured $x$-ray power at $Z$ has been more modest $\left(~ Z 200 \times 10^{12} \mathrm{~W}\right.$ with $\mathrm{x}$-ray yields $\gtrsim 2 \times 10^{6} \mathrm{~J}$ into $4 \pi \mathrm{sr}$ ) and represents a lower brightness temperature $[9,15,18,25]$. (The $Z$ accelerator was updated and renamed ZR in 2007.)

In this article, we focus our attention on continuum spectra from a $z$ pinch, assuming (a) that the emitted spectrum $S(E, t)$ may be-but need not be-Planckian; (b) that the high-energy tail of this spectrum is at least asymptotically bounded by a $250-\mathrm{eV}$ Planckian, just estimated; and (c) that negligible radiated flux is associated with line spectra [7] (cf. Sec. IV and Pt. 2: Sec. III).
Raw XRD voltage signals $V_{i}(t)$ for $Z$-shot 165 (a typical, bare, tungsten, $z$-pinch experiment) are shown in Fig. 3. At peak, the noise-to-signal ratio (NSR) was $\sim 1 \%$ in channels $1-4$ and $\sim 5 \%$ in channel 5 . Within $\sim 3$ ns of peak signal, a $(\mathrm{NSR}) \lessgtr 10 \%$ is still a reasonable bound for all the channels. The corresponding reduced channel data, $D_{1}(t), \ldots, D_{5}(t)$, in Eq. (1) are similar to these traces because the channel-wise solid angles were identical.

From such time-dependent measurements $D_{i}(t)$ we wish to invert Eq. (1), extracting enough information about $S(E, t)$ to estimate the spectrally integrated, timedependent x-ray flux [28]. There are, in fact, several measures of interest, of which we define

$$
\mathcal{F}(t) \equiv \int_{0}^{E_{\mathrm{MAX}}} S(E, t) d E
$$

and

TABLE I. A priori information concerning source x-ray spectra and response functions for the filtered-XRD diagnostic at the $Z$ accelerator.

\begin{tabular}{|c|c|c|c|}
\hline Assumption & Description & Specific effects & Section cited \\
\hline 1 & $\begin{array}{l}\text { Eq. (1) connects data, spectra, and noise } \\
\text { at each time } t \text {. }\end{array}$ & $\begin{array}{l}\text { Data is linear with the spectral components } \\
\text { and without threshold or saturation effects. } \\
\text { Error sources are systematic or random. }\end{array}$ & Pt. 1: II A, Pt. 2: II \\
\hline 2 & $\begin{array}{l}R_{i}(E) \text { and } S(E, t) \text { are real and square } \\
\text { integrable over their espective domains: } \\
\text { i.e., } \int R_{i}^{2}(E) d E \text { is finite over }\left[0, E_{\mathrm{MAX}}\right] \text {, } \\
\text { as is } \int S^{2}(E, t) d E d t \text { over }\left[0, E_{\mathrm{MAX}}\right] \times \\
{\left[0, t_{\mathrm{END}}\right] \text {, where } t_{\mathrm{END}} \text { marks the termina- }} \\
\text { tion of data. }\end{array}$ & $\begin{array}{l}R_{i} \text { and } S(E, t) \text { at fixed } t \text { belong to the real } \\
\text { Hilbert space } \mathrm{L}_{2} \text { of square-integrable, real } \\
\text { functions, defined on }\left[0, E_{\mathrm{MAX}}\right] \text {. Linear } \\
\text { functionals and operators may be defined } \\
\text { on this space, but certain unbounded behav- } \\
\text { ior is excluded. The total energy deposited } \\
\text { in the diagnostic during irradiation is finite. }\end{array}$ & $\begin{array}{l}\text { Pt. 1: II D, Pt. 1: III C, } \\
\text { Pt. 1: IV, Pt. 2: III }\end{array}$ \\
\hline 3 & $R_{i}, S \geq 0$ for $i=1, \ldots, N$ & $\begin{array}{l}\text { Noise-free data are non-negative. Any } \\
\text { negative-valued real data must, therefore, } \\
\text { come from the noise terms, } \varepsilon_{i} \text {, in Eq. (1). }\end{array}$ & Pt. 1: IV, Pt. 2: III \\
\hline 4 & $\begin{array}{l}S(E) \text { extends }{ }^{\mathrm{a}} \text { over the } R_{i} \text { 's but not sig- } \\
\text { nificantly beyond }\end{array}$ & $\begin{array}{l}\text { An effective unfold interval }[\Delta E]= \\
{\left[E_{\mathrm{LO}}, E_{\mathrm{HI}}\right]=[137,2300] \mathrm{eV} \text { is identified, }} \\
\text { outside of which } S_{\text {unfold }} \equiv 0\end{array}$ & Pt. 1: III A. Pt. 2: III \\
\hline 5 & $\begin{array}{l}S(E) \text { is broad and nearly continuous }{ }^{\text {b }} \\
\text { over the } R_{i} \text { 's }\end{array}$ & $\begin{array}{l}{[\Delta E] \text { can be partitioned by the response }} \\
\text { functions and histograms may be used to } \\
\text { approximate } S_{\text {unfold }}\end{array}$ & Pt. 1: III D \\
\hline 6 & $\begin{array}{l}\text { Depending on the spectral temperature, } \\
\text { Planckian spectra are feasible, but insuf- } \\
\text { ficient to represent all admissible, con- } \\
\text { tinuum source spectra }{ }^{\text {c }}\end{array}$ & $\begin{array}{l}\text { Test cases for Eq. (1) and the unfold proce- } \\
\text { dure are provided. }\end{array}$ & Pt. 1: III A, IV \\
\hline 7 & $\begin{array}{l}250 \mathrm{eV} \text { is the maximum brightness tem- } \\
\text { perature expected }\end{array}$ & $\begin{array}{l}\text { Bounds the unfold interval }[\Delta E] \text { at high } \\
\text { x-ray energies }\end{array}$ & Pt. 1: II B, Pt. 1: III A \\
\hline 8 & $\begin{array}{l}\text { Perturbations } \varepsilon_{i} \text { may be described as the } \\
\text { sum of independent perturbations in the } \\
\text { data and the responses. }\end{array}$ & Error propagation & Pt. 2: II \\
\hline
\end{tabular}

\footnotetext{
${ }^{a} S$ is thus assumed not to be concentrated over the tail regions of the responses. In principle, one could field multiple diagnostics with differing responses so that this expectation would be true of some response set.

${ }^{\mathrm{b}} S$ is thus without appreciable structure compared to the response functions.

${ }^{c}$ Hence Planckian spectra will here be considered as reasonable approximations but not as constraints on $S_{\text {unfold }}$.
} 


$$
\mathcal{F}_{[\Delta E]}(t) \equiv \int_{E_{\mathrm{LO}}}^{E_{\mathrm{HI}}} S(E, t) d E
$$

where $\mathcal{F}$ represents the total incident x-ray flux of $S$ (for $E_{\mathrm{MAX}}$ sufficiently large) and $\mathcal{F}_{[\Delta E]}$ is the flux of $S$ in a yet-to-be-specified subinterval, $[\Delta E] \equiv\left[E_{\mathrm{LO}}, E_{\mathrm{HI}}\right]$, of $\left[0, E_{\mathrm{MAX}}\right]$ [cf. Eq. (1)]. Both these measures are functionals of $S$ (Pt. 2: Table I), as is a third x-ray flux measure, $\mathcal{F}_{\text {unfold }}$, defined below in Eq. (15).

\section{Inversion difficulties and mitigation for Fredholm integral equations of the first kind}

With the noise terms $\varepsilon_{i}$ ignored, the mathematical model postulated in Eq. (1) at each time step may be rearranged as

$$
\int_{a}^{b} R_{i}(x) f(x) d x=y_{i} \quad(i=1, \ldots, N),
$$

known as an inhomogeneous, discrete, Fredholm integral equation of the first kind [49,53,54,63,73-76]; the order of variables emphasizes that the $y_{i}$ 's and $R_{i}$ 's are given and $f$ is sought. In general, these quantities have no physical significance, although we evocatively refer to the $R_{i}$ 's as responses and the $y_{i}$ 's as channel data. However, we call the $f$ 's spectral functions, not spectra $S$ (which are further constrained by Table I).

Equation (5) has been much studied [35,49-54,6163,73-93]. Viewed as a linear mapping $\mathcal{M}$ from an infinite-dimensional space of real functions $f(x)$ into an $N$-dimensional space of real data vectors $\left(y_{1}, \ldots, y_{N}\right)$ [cf. Pt. 2: Sec. III], it associates each $f$ with one data vector, as long as the integrals in Eq. (5) exist. However, due to the disparity in dimension between these functions and vectors, $\mathcal{M}$ is perforce a many-to-one mapping; that is, many distinct $f$ 's are associated with at least some vectors $\left(y_{1}, \ldots, y_{N}\right)$ [84]. Hence, there is no necessary association of arbitrary $\left(y_{1}, \ldots, y_{N}\right)$ with a unique $f$ and thus no general inverse mapping $\mathcal{M}^{-1}$. In the mathematical literature, inverse problems suffering from nonuniqueness (among other maladies) are diagnosed as ill posed $[49,52,63,80]$.

If the nature of $\mathcal{M}$ in Eqs. (5) and (1) is ignored and an inversion $f(x)$ is nevertheless attempted for given data, a number of redoubtable mathematical pathologies may be encountered $[49,52,63]$. These difficulties include issues already noted (Sec. I) plus the following: (1) Since only $R_{i}$-weighted moments of $f(x)$ appear in Eq. (5) —not $f(x)$ itself-fine details in $f(x)$ can be obliterated (e.g. averaged out or distorted) in $\left(y_{1}, \ldots, y_{N}\right)$ [49]. (2) The many-to-one nature of $\mathcal{M}$ implies the existence of nontrivial functions $f_{0}(x)[61,62,94]$ satisfying

$$
\int_{a}^{b} R_{i}(x) f_{0}(x) d E \equiv 0
$$

for all $i=1, \ldots, N$; we call these null functions (or invisible functions [51]). If the $R_{i}(x)$ 's in Eqs. (5) and (6) are all non-negative, such null functions must either oscillate in sign or be zero valued. It follows from Eqs. (5) and (6) that if $f(x)$ is mapped into vector $\left(y_{1}, \ldots, y_{N}\right)$, then so is $f(x)+$ $\alpha f_{0}(x)$ for arbitrarily large scalars $\alpha$. What is more, $f_{0}(x)$ need have no relation at all to $f(x)$. Thus, even exact knowledge of the functions $R_{i}(x)$ and the vectors $\left(y_{1}, \ldots, y_{N}\right)$ in Eq. (5) is insufficient to distinguish $f(x)$ from $f(x)+\alpha f_{0}(x)$ in an inversion process [49]. Left unaddressed, this pathology can lead to distinct inversions, (say) $f_{\text {unfold }}^{(1)}(x)$ and $f_{\text {unfold }}^{(2)}(x)$ from different unfold algorithms, that bear no resemblance to one another, nor to $f(x)$, and yet identically satisfy Eq. (5) $[49,95]$. (3) A complementary pathology obtains if some inconsistency exists among the data, responses, and spectral functions. There may then be unrealistic associations, or no associations at all [94], between a given data vector $\left(y_{1}, \ldots, y_{N}\right)$ and any $f(x)$. Major causes of inconsistency include the following: (a) A prescribed parametric form of $f(x)$ may be incorrect; e.g., Planckian spectra might be erroneously assumed for data actually generated by line spectra. (b) The responses $R_{i}(x)$ substituted in Eq. (5) may not be the functions that generate $\left(y_{1}, \ldots, y_{N}\right)$. (c) Some $R_{i}(x)$ 's may be redundant; thus, of $N$ pieces of data measured, only $M(<N)$ represent independent information about $f(x)$, and arbitrary $\left(y_{1}, \ldots, y_{N}\right)$ cannot be directly addressed by $\mathcal{M}[50,51,61,62]$. (4) Even marginally independent $R_{i}(x)$ 's $[49,51,62,63]$ may still overwhelm a spectral inversion by uncontrollably amplifying computational and experimental noise. (The associated inversion matrices are then termed ill conditioned $[49,62,63,82,96,97])$.

In summary, unfold algorithms for Eqs. (5) and (1) by themselves are bedeviled by uncontrolled null spectra, inconsistencies, instabilities, and sensitivity to error. Numerical tests have been devised to quantify the potential of a given unfold problem for such pathological behavior (cf. Sec. III, Appendix D, and [82,83,96,97]).

Partial recovery of $f(x)$ in Eq. (5) may, however, be possible if one can inject some trustworthy information about $f(x)$ into the problem-in addition to the measured data and the response functions. Such information is called a priori information and translates into constraints $[49,51,53,62,63,80,83]$ on an approximate reconstruction, $f_{\text {unfold }}(x)$, of $f(x)$. The goal is to suppress the pathologies just noted, while recovering $f(x)$ in sufficient detail for some diagnostic purpose.

A priori information [51] may derive from theoretical considerations or other cofielded diagnostics and encompasses many forms of constraint. For example, such extra information might prescribe some particular parametric expression for $f_{\text {unfold }}(x)$, imply numerical bounds on $f_{\text {unfold }}(x)$ and its derivatives, or suggest a least-squares minimization criterion to "resolve [52]" Eqs. (1) and (5) -as opposed to an exact solution. In fact, we have already presented an energy argument (Sec. II B) which bounds the brightness temperature for bare $z$ pinches on the $Z$ accelerator to $\lesssim 250 \mathrm{eV}$. This information is used below 
(Sec. III A) to shorten the domain of $S_{\text {unfold }}$ relative to the larger energy interval $\left[0, E_{\mathrm{MAX}}\right]$, suggested by Fig. 1. More sophisticated constraints may be imposed by simultaneously fitting the measured data while optimizing some other spectral function, an approach (called regularization) that generally leads to a tradeoff between spectral resolution and overall uncertainties in the reconstruction $[49,50,62,74,80,81]$. This article is not a general review of unfolding techniques, which constitute a major field of mathematical study; the interested reader may consult [35,49-54,61-63,77-82,85-89].

\section{Blackbody spectra as parametric spectral functions for $z$-pinch plasmas}

As noted above, the focus of this article is the $\mathrm{x}$-ray flux, $\int S(E, t) d E$, incident on the $Z$ diagnostic, and we wish to impose as little a priori information about $S(E, t)$ as necessary.

One possible course is to prescribe a blackbody (Planckian) spectrum $S_{b b}(E, T)[5,7,37,98]$ of the form

$$
S_{b b}(E, T)=K E^{3}\left(e^{E / T}-1\right)^{-1},
$$

for $S_{\text {unfold }}(E, t)$. (Here, $T$ is a time-dependent parameter and $K=2 c^{-2} h^{-3} \approx 5 \mathrm{~kW} \mathrm{sr}^{-1} \mathrm{~cm}^{-2} \mathrm{eV}^{-4}$ for $E$ and $T$ in $\mathrm{eV}$.) Then, in principle, a comparison of simulated noisefree data $\left[d_{1}(T), \ldots, d_{N}(T)\right]$,

$$
d_{i}(T)=\int_{0}^{E_{\mathrm{MAX}}} R_{i}(E) S_{b b}(E, T) d E,
$$

with measured data, $\left[D_{1}(t), \ldots, D_{N}(t)\right]$, estimates $T(t)$ [99]. (This approach is useful when the parametrization of $S(E, t)$ is well known and the fit parameters are few [92,99-103].)

Figure 4 makes such a comparison for the $Z$ diagnostic. The broken lines represent simulated data, $d_{i}(T)$, generated from Planckian spectra, $S_{b b}(E, 150 \leq T \leq 225 \mathrm{eV})$, and the responses, $R_{1 b}, \ldots, R_{5 b}$, in Fig. 2. The solid dots $D_{i}$ are peak data taken during $Z$-shot 165 (Fig. 3) with this calibration and have been uniformly scaled to force $D_{1} \equiv d_{1}(200 \mathrm{eV})$. (Normalization is used here because the $Z$ diagnostic is not spatially resolving, cf. Pt. 2: Sec. II C, where plasma dimensions from imaging diagnostics are included.)

It is clear from Fig. 4 that the $D_{i}$ are, in fact, most similar in shape to the scaled $d_{i}(T=200 \mathrm{eV})$. But, one also notes distinct disagreement at channel 3, the response function of which appears to be both stable and reproducible (Fig. 2). If this calibration of $R_{3 b}$ is accepted, then one may infer that the actual peak spectrum in this shot differed from a simple Planckian spectrum, $S_{b b}(E, T)$. Spatial variations in opacity and temperature, for example, can produce nonPlanckian spectra.

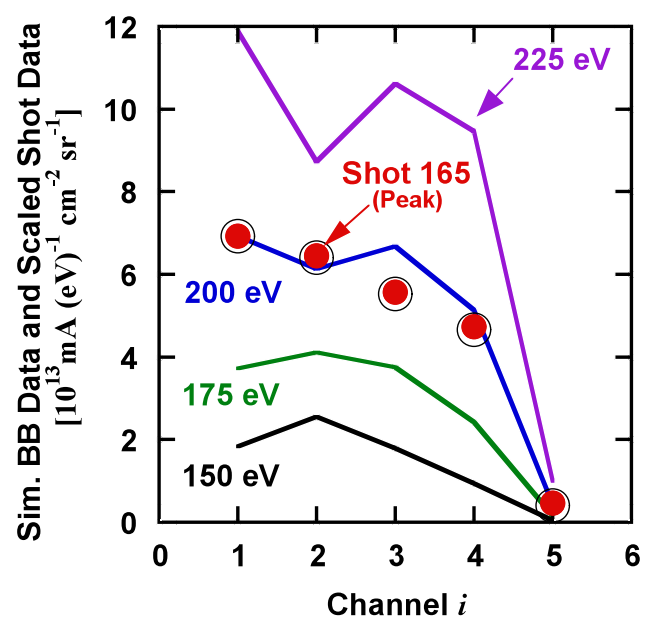

FIG. 4. Comparison of simulated Planckian data with scaled peak experimental data from $Z$-shot 165 . The simulated profiles, $d_{i}(T)$ vs $i$, were constructed from responses $R_{1 b}, \ldots, R_{5 b}$ (Fig. 2) and Planckian spectra $S_{b b}(E, 150 \leq T \leq 250 \mathrm{eV})$. The experimental profiles, $D_{i}$ vs $i$, were obtained in two ways: the solid points correspond to peak values of $V_{i}(t)$ in Fig. 3, while the open circles derive from interpolated values of $V_{i}$ when $V_{1}(t)$ peaks. The two $D_{i}$-vs- $i$ profiles have been independently scaled to coincide with channel 1 of $d_{i}(200 \mathrm{eV})$ vs $i$.

\section{E. A priori assumptions about $S(E, t)$ adopted here}

Given the comparison in Fig. 4, it seems prudent to be less explicit about the parametric form of spectra emitted by $z$-pinch plasmas. Table I summarizes the general $a$ priori assumptions about spectra considered here. The effect of each assumption on $S$ is identified in the table, together with pertinent notes. To reiterate, spectra that at least approximately follow these assumptions are denoted by $S$, with reconstruction $S_{\text {unfold }}$ (Secs. II, III, IV, and V); the corresponding notations, $f$ and $f_{\text {unfold }}$, are used for more general spectral functions (Pt. 2).

\section{DEVELOPMENT OF THE UNFOLD ALGORITHM FOR THE $Z$ DIAGNOSTIC}

This section illustrates the general strategy $[49,51]$ used to attack the inversion problem posed in Eqs. (1) and (5) for the $Z$ diagnostic. Unfolding techniques are generally problem dependent [49-52,80,81], and several algorithms may be valid. The initial step in this plan probed the response functions for the actual spectral information encoded: an effective energy domain was determined for $S$; channel redundancy was assessed; and the spectral resolution of the responses was estimated. This information, combined with further physical constraints on $S$, then suggested a feasible reconstruction $S_{\text {unfold }}$ for the desired estimate of x-ray flux. Equation (1) was then reformulated as an $N \times N$ problem and inverted numerically. The assumed constraints (Table I) were found sufficient to control the inherent ill posedness of the inversion process. An upper bound to 
error propagation was estimated. The final unfold algorithm is given in Eqs. (20) and (21). (The principal equations are also summarized in Pt. 2: Sec. I.)

\section{A. Defining an effective unfold $x$-ray energy domain: $[\Delta E]$}

Since Eq. (1) integrates $R_{i}(E) S(E)$ over a wide enough domain to account for nearly all soft-x-ray interactions in the $Z$ diagnostic (Fig. 1), one might think that a reconstruction, $S_{\text {unfold }}(E)$, must cover the same interval, e.g., $\left[0, E_{\mathrm{MAX}} \geqslant 5 \mathrm{keV}\right]$ in Fig. 1. However, often a smaller unfold domain, $[\Delta E] \equiv\left[E_{\mathrm{LO}}, E_{\mathrm{HI}}\right] \subset\left[0, E_{\mathrm{MAX}}\right]$, is appropriate for $S_{\text {unfold }}$ if the responses mostly detect x rays over a narrower energy range and if typical spectra are ignorable for $E \ll E_{\mathrm{MAX}}$ - a process analogous to cropping a photographic image. In fact, contracting the unfold domain limits some pathological unfold behaviors. For example, if all the $R_{i} \rightarrow 0$ in some local x-ray region, then $S_{\text {unfold }}(E)$ is untied to channel data $D_{i}$ in that region [cf. Eq. (6)] and can assume arbitrary values, unless further constrained. [A corresponding abnormality can occur if $S_{\text {unfold }}(E)$ is unconstrained where one expects $S(E) \rightarrow 0$ ]. Sometimes these pitfalls can be avoided by limiting $S_{\text {unfold }}(E)$ to a subinterval $\left[E_{\mathrm{LO}}, E_{\mathrm{HI}}\right]$ of $\left[0, E_{\mathrm{MAX}}\right]$. (Effects of misjudging $[\Delta E]$ are noted in Sec. IV and Pt. 2: Sec. III.)

Since neither the $R_{i}$ 's of the $Z$ diagnostic nor the expected $S(E)$ define precise thresholds, $E_{\mathrm{LO}}$ and $E_{\mathrm{HI}}$, for the unfold interval $[\Delta E]$, it is reasonable to look for integral criteria. For this we chose a sequence of Planckian spectra $S_{b b}(E, 60 \mathrm{eV} \leq T \leq 250 \mathrm{eV})$ and simulated x-ray data with each. We then required $E_{\mathrm{LO}}$ and $E_{\mathrm{HI}}$ to be chosen so that within $\left[E_{\mathrm{LO}}, E_{\mathrm{HI}}\right]$ each $S_{b b}(E, T)$ accounted at least for a prescribed fraction $(1-\alpha)$ of the total channel data due to $\left[0, E_{\mathrm{MAX}}\right]$. We chose $\alpha=5 \%$, so that $\left[E_{\mathrm{LO}}, E_{\mathrm{HI}}\right]$ is analogous to a $95 \%$ statistical tolerance interval of width $\Delta E=E_{\mathrm{HI}}-E_{\mathrm{LO}}[104,105]$. One finds that $E_{\mathrm{LO}}$ and $E_{\mathrm{HI}}$ can be chosen independently, and that neither depends on the spectral shape within $[\Delta E]$ (Appendix C). The result,

$$
\left[E_{\mathrm{LO}}, E_{\mathrm{HI}}\right] \equiv[137 \mathrm{eV}, 2300 \mathrm{eV}] \equiv[\Delta E],
$$

satisfies our fractional-data requirement for all the selected Planckian spectra.

Figure 5 places $[\Delta E]$ within the context of $60-\mathrm{eV}$ and 250-eV, Planckian spectra. The dashed curves are normalized: $\underline{S}_{b b}(E, T) \equiv S_{b b}(E, T)\left[\int_{0}^{E_{\mathrm{MAX}}} S_{b b}\left(E^{\prime}, T\right) d E^{\prime}\right]^{-1}$. The solid lines indicate cumulative integrals, $I(E, T)=$ $\int_{0}^{E} \underline{S}_{b b}\left(E^{\prime}, T\right) d E^{\prime}$, representing the fractional flux for $E \leq$ $E_{\mathrm{MAX}}$. (For convenience in this example, numerical integration was actually terminated at 1.2 and $5 \mathrm{keV}$ for $T=$ 60 and $250 \mathrm{eV}$, respectively; extended computation altered the result by $<0.1 \%$.) One sees that $\sim 25 \%$ of the flux of $S_{b b}(E, 60 \mathrm{eV})$ lies below $E_{\mathrm{LO}}(137 \mathrm{eV})$ and is largely undetected in the $Z$ diagnostic (Appendix E, Fig. 13). At

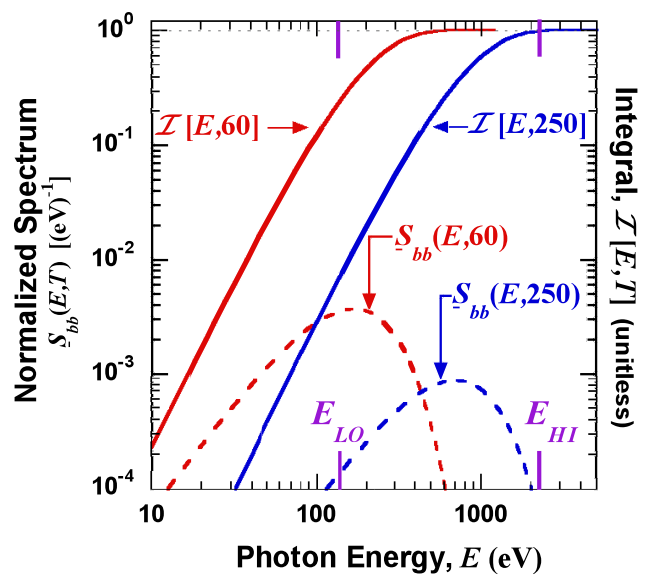

FIG. 5. Normalized spectral shapes and cumulative shape integrals for Planckian spectra of temperatures $T=60 \mathrm{eV}$ and $250 \mathrm{eV}$. The dashed curves (left axis) are normalized Planckian shape functions, $\underline{S}_{b b}(E, T)$. The solid curves [right axis] show the corresponding cumulative integrals, $I[E, T]=\int_{0}^{E} \underline{S}_{b b}\left(E^{\prime}, T\right) d E^{\prime}$ for $E \leq E_{\mathrm{MAX}}$, where $I\left[E_{\mathrm{MAX}}, T\right] \equiv 1$. The unfold interval, $[\Delta E]=\left[E_{\mathrm{LO}}, E_{\mathrm{HI}}\right]=[137 \mathrm{eV}, 2300 \mathrm{eV}]$ is indicated. (Both ordinate axes share the same numerical scale, but different units.)

high energy, only $\sim 2 \%$ of $S_{b b}(E, 250 \mathrm{eV})$ lies above $E_{\mathrm{HI}}(2300 \mathrm{eV})$.

\section{B. Assessing the independence of the $\boldsymbol{R}_{i}(E)$ 's}

The $N$ response functions for the $Z$ diagnostic were next probed for linear dependence (Pt. 2: Table I). One can show that if (say) $N-M$ of these are dependent [i.e., $R_{k}(E)=$ $\left.\sum_{i=1}^{M} \beta_{k, i} R_{i}(E), M<k \leq N\right]$, then the corresponding X-ray data $d_{i}$ are redundant and exhibit a channel-wise pattern: $\left(d_{1}, \ldots, d_{M}, \sum_{i=1}^{M} \beta_{M+1, i} d_{i}, \ldots, \sum_{i=1}^{M} \beta_{N, i} d_{i}\right)$ (Appendix D). But, the measured data $\left(D_{i}=d_{i}+\varepsilon_{i}\right)$ need not follow this pattern because they include perturbations $\varepsilon_{i}$ that may be unrelated to the responses. Thus, in general, there is no spectrum $S(E)$ that uniquely and exactly satisfies Eq. (1) for such dependent responses ([50,51,61,62], Appendix D). On the other hand, if the responses are linearly independent, one can demonstrate at least one exact solution to Eq. (1) for arbitrary measured $D_{i}$ (Appendix D, Sec. III E). The absence or presence of linear dependence thus suggests whether an exact solution or a solution based on some other criteria (e.g., least squares $[14,49])$ is appropriate.

The response set $\left\{R_{i}(E)\right\}_{i=1}^{N}$ was tested for dependence over domain $[a, b]$ by examining the Gram matrix $\mathbb{G}[a, b]$ for singularity $[52,53,62,106-112]$, where

$$
(\mathbb{G}[a, b])_{i j}=G_{i j} \equiv \int_{a}^{b} R_{i}(E) R_{j}(E) d E .
$$

$\mathbb{G}$ is a real, symmetric, non-negative, $N \times N$ matrix for the $R_{i}(E)$ 's considered here. For example, $\mathbb{G}[137 \mathrm{eV}, 2300 \mathrm{eV}]$ for responses $R_{1}, \ldots, R_{5}$ in Fig. 1 is 


$$
\begin{aligned}
\mathbb{G}[137 \mathrm{eV}, 2300 \mathrm{eV}]= & \left(321 \frac{\mathrm{pA}^{2} \mathrm{eV}}{\mathrm{W}^{2}}\right) \\
& \times\left(\begin{array}{cccccc}
25 & 4 & 8 & 20 & 3 \\
* & 11 & 1 & 3 & 1 \\
* & * & 12 & 9 & 1 \\
* & * & * & 19 & 3 \\
* & * & * & * & 1
\end{array}\right),
\end{aligned}
$$

where, for clarity, a common scalar has been factored out and symmetric elements are not displayed. (The other responses in Fig. 2 and wider integration intervals yield similar results.)

$\mathbb{G}$ was then factored by the method of singular-value decomposition (SVD) $[49,51,61,74,75,82,83,96,97]$ to extract (Appendix D) the $N$ singular values, $g_{1} \geq g_{2} \geq$ $\cdots \geq g_{N} \geq 0[49,54,76,82,84,96,97,106,113]$, for which $|\operatorname{det}(\mathbb{G})|=\Pi_{i=1}^{N} g_{i}[114]$. $\mathbb{G}$ is exactly singular if any $g_{k} \equiv$ 0 . No null values were found for these Gram matrices.

In numerical contexts, singular values $g_{k} \cong 0$ indicate approximate singularity (i.e., ill conditioning) of $\mathbb{G}$ and can still be troublesome for computational stability and error propagation (cf. Sec. IVA). Two simple tests were applied. For the first, $\left(g_{k} / g_{1}\right)$ vs $k$ was plotted and scrutinized for a sharp drop after $M<N$ terms: by convention a discontinuity of the order of computational round-off error $(\leq 3 \times$ $10^{-7}$ for a $5 \times 5$ matrix in single precision (Appendix D, $[74,82]$ ) indicates ill conditioning. Alternatively, the condition number $[49,51,74,75,82,83,96,97]$, cond $(\mathbb{G})=$

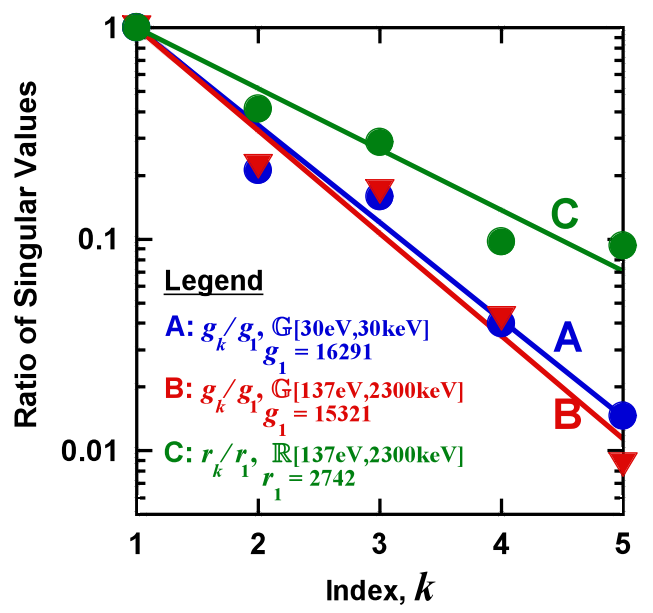

FIG. 6. Ratios of singular values (SV's) for the Gram matrix $\mathbb{G}$ and response matrix $\mathbb{R}$. The points labeled A (blue) and B (red) are the SV ratios, $g_{k} / g_{1}$, of $\mathbb{G}$ evaluated over intervals, [30 eV, $30 \mathrm{keV}$ ] and [137 eV, $2300 \mathrm{eV}$ ], respectively, while points $\mathrm{C}$ are the $\mathrm{SV}$ ratios, $r_{k} / r_{1}$, of $\mathbb{R}$ for $[137 \mathrm{eV}, 2300 \mathrm{eV}]$. The legend notes the largest SV's, $g_{1}$ and $r_{1}$, for each set of points. (The solid lines are least-squares fits to guide the eye.) The condition number, cond $(\mathbb{G})=g_{1} / g_{5}$, ranges from $68-116$, depending on the evaluation interval; and $\operatorname{cond}(\mathbb{R})=r_{1} / r_{5}$ is $\sim 11$.
$\left(g_{N} / g_{1}\right)^{-1}$, indicates ill conditioning for large values ( $\gtrsim 3 \times 10^{6}$ [74], Appendix D).

Figure 6 shows $\left(g_{k} / g_{1}\right)$ vs $k$ for the Gram matrices $\mathbb{G}$ $[137 \mathrm{eV}, 2300 \mathrm{eV}]$ and $\mathbb{G}[30 \mathrm{eV}, 30 \mathrm{keV}]$, noted above. No large drops are visible; in fact, the smallest ratios of singular values are $0.0086(\cong 1 / 116)$ and $0.0146(\cong$ $1 / 68)$, respectively. Hence, at worst, $\operatorname{cond}(\mathbb{G})$ is $\sim 116$, well below the threshold for ill conditioning.

The above analysis, therefore, shows that the responses of the $Z$ diagnostic in Figs. 1 and 2 are linearly independent over a wide domain of $\mathrm{x}$-ray energies.

\section{Estimating the spectral resolution of the $\boldsymbol{R}_{i}$ 's}

Although linearly independent, the response functions of the $Z$ diagnostic nevertheless overlap considerably. Thus, our third assessment probed for $\mathrm{x}$-ray discriminating regions, defined collectively, which may span the unfold interval $[\Delta E]$. The result is relevant for approximating $S$.

A useful method of defining resolution is the BackusGilbert (BG) technique [115-117]. This approach has been extensively examined $[49,50,53,61-63,74,82,112]$ and is not duplicated here. It (a) determines linear combinations of the given $R_{i}$ 's that represent narrower "hybrid" responses than the $R_{i}$ 's alone and (b) associates the defined "widths" of these hybrids with spectral resolution regions or bins. The BG method requires independent $R_{i}$ 's in $[\Delta E]$ but assumes no spectral shape for $S$.

A detailed $\mathrm{BG}$ analysis of responses $R_{1}, \ldots, R_{5}$ in Fig. 1 has been reported in Ref. [55]. Table II shows the five resolution bins thus obtained. As suggested by Fig. 1, BG supports three, low x-ray-energy bins, defined by $E_{\mathrm{LO}}(137 \mathrm{eV})$ and the characteristic x-ray edges (284, 513, and $1020 \mathrm{eV}$ ) but also adds two high-energy bins below the upper x-ray limit $E_{\mathrm{HI}}(2300 \mathrm{eV})$. For this set of $(N=5)$ response functions, $[\Delta E]$ can thus be effectively subdivided into $(M=N)$ contiguous resolution bins. Table II lists other binwise characteristics of this partition; the $N$ bin widths $\Delta E_{j}$ will sometimes be regarded as components of a fixed vector, $\Delta \mathbf{E}$.

TABLE II. Resolution bins for response functions $R_{1}, \ldots, R_{5}$ (Fig. 1) as estimated by the Backus-Gilbert method [115-117]. The $j$ th bin is variously denoted as $\left[E_{j}, E_{j}+1\right]$ and $\left[\Delta E_{j}\right]$ in the text; its length is $\Delta E_{j}=E_{j+1}-E_{j}$. The average x-ray energy in the $j$ th bin is denoted as $\bar{E}_{j}=\left(E_{j+1}+E_{j}\right) / 2$. The spectral resolution, $\bar{E}_{j} / \Delta E_{j}$, is also noted.

\begin{tabular}{lcllll}
\hline \hline Bin $j \rightarrow$ & 1 & 2 & 3 & 4 & 5 \\
\hline$E_{j}(\mathrm{eV})$ & 137 & 284 & 513 & 1020 & 1500 \\
$E_{j+1}(\mathrm{eV})$ & 284 & 513 & 1020 & 1500 & 2300 \\
$\bar{E}_{j}(\mathrm{eV})$ & 211 & 399 & 767 & 1260 & 1900 \\
$\Delta E_{j}(\mathrm{eV})$ & 147 & 229 & 507 & 480 & 800 \\
$\bar{E}_{j} / \Delta E_{j}$ & 1.4 & 1.7 & 1.5 & 2.6 & 2.4 \\
\hline \hline
\end{tabular}




\section{Defining an approximation $S_{\text {unfold }}$ to the spectral source $S$ and the unfolded flux $\mathcal{F}_{\text {unfold }}$}

Solutions to integral-differential equations are often obtained as infinite-series expansions [73]. Although dimensional incompatibility precludes such an approach to Eqs. (1) and (5), it is reasonable to consider a finite expansion,

$$
S_{\text {unfold }}(E) \equiv \sum_{j=1}^{M} S_{j} B_{j}(E),
$$

as an approximation to $S(E)$, where the $B_{j}$ 's are basis functions. The coefficients $S_{j}$ are to be determined from the data vector $\left(D_{1}, \ldots, D_{N}\right)$ plus available a priori information about the source $S(E)$. Such projection or Galerkin methods are well documented [50,52,54,75,80-82,85,112]. (For convenience, we have suppressed the time variable $t$.)

Supported by the previous subsection, we chose $M=$ $N=5$ expansion terms. Moreover, to estimate x-ray flux we were not particularly interested in the spectral details within resolution bins $\Delta E_{j}$, and so we chose first-order Bsplines as basis functions $B_{j}[118]$ :

$$
B_{j}(E)= \begin{cases}1 & \text { if } E_{j} \leq E<E_{j+1} \\ 0 & \text { otherwise }\end{cases}
$$

where the $E_{j}$ 's are listed in Table II. These $B_{j}$ 's are orthogonal (but non-normalized) and zero outside $[\Delta E]$.

The function $S_{\text {unfold }}(E)$ in Eq. (12) is thus a histogram approximation to $S(E)$, which is consistent with the demonstrated independence of the $R_{i}$ 's and their spectral resolution, a priori assumptions about $S(E)$, and the effective domain $[\Delta E]$. This representation also suppresses null solutions $S_{0}(E)$ of Eq. (1) of finer detail than $\Delta E_{j}$, and constitutes an elementary form of regularization $[49,50,80,81,85,113]$. But, more importantly, $S_{\text {unfold }}(E)$ includes only enough functional detail to estimate the x-ray flux in $\left[\Delta E_{j}\right]$ if $S(E)$ is well behaved. To see this, suppose $S(E)$ can be represented in $\left[\Delta E_{j}\right]$ by a Taylor expansion about its midpoint $\bar{E}_{j}$ : in general, $S\left(\bar{E}_{j}\right)$ and all its derivatives are needed. The flux in $\left[\Delta E_{j}\right]$, however, requires only even derivatives (Appendix E):

$$
\begin{aligned}
\int_{E_{j}}^{E_{j+1}} S(E) d E \cong & S\left(\bar{E}_{j}\right) \Delta E_{j}\left\{1+\frac{1}{24} \frac{S^{\prime \prime}\left(\bar{E}_{j}\right)\left(\Delta E_{j}\right)^{2}}{S\left(\bar{E}_{j}\right)}\right. \\
& \left.+\frac{O\left[\left(\Delta E_{j}\right)^{4}\right]}{S\left(\bar{E}_{j}\right)}\right\},
\end{aligned}
$$

where $S\left(\bar{E}_{j}\right) \neq 0$. Hence, a zeroth order estimate of $\int S(E) d E$ corresponds to the histogram approximation $S_{\text {unfold }}(E)$ from Eqs. (12) and (13) (cf. Sec. III H).

Different response functions and constraints may suggest other representations $[49,50]$ of $S_{\text {unfold }}(E)$. For example, a set of orthogonal polynomials defined on $[\Delta E]$ or even a sequence of Planckian functions [Eq. (7)] with prescribed temperatures may qualify $[14,49]$. Knauer [119] and others $[103,120]$ have successfully used higher-order B-splines for filtered-detector arrays.

Given $S_{\text {unfold }}(E)$, the unfold flux estimate $\mathcal{F}_{\text {unfold }}$ was defined as

$$
\begin{aligned}
\mathcal{F}_{\text {unfold }} & \equiv \int_{0}^{E_{\mathrm{MAX}}} S_{\text {unfold }}(E) d E=\int_{0}^{E_{\mathrm{MAX}}} \sum_{j=1}^{N} S_{j} B_{j}(E) d E \\
& =\sum_{j=1}^{N=5} S_{j} \Delta E_{j}
\end{aligned}
$$

to be compared with $\mathcal{F}$ and $\mathcal{F}_{[\Delta E]}$ [Eqs. (3) and (4)]. Equation (15) also applies to time-dependent quantities and general spectral functions $f(E)$.

Some spectral shapes $S(E)$ are, however, ill approximated by the basis functions of Eq. (13). In particular, sources $S(E)$ showing (a) dominant structure or (b) significant values outside $[\Delta E]$ prove problematic (cf. Pt. 2: Sec. III B 2). A quantitative measure of how closely these $B_{j}$ 's approximate arbitrary $S(E)$ is noted in [121] and in Figs. 8 and 9.

\section{E. Reformulating Eq. (1) with $S_{\text {unfold }}(E)$}

The approximation $S_{\text {unfold }}$ was inserted into the channeldata model to convert Eq. (1) into a matrix equation [49]. It is simplest to substitute $S_{\text {unfold }}$ into the left-hand side of Eq. (1) and require exact equality with the measured channel data $D_{i}$ :

$$
\begin{aligned}
\int_{E_{\mathrm{LO}}}^{E_{H I}} R_{i}(E) S_{\text {unfold }}(E, t) d E= & D_{i}(t) \\
= & \int_{0}^{E_{\mathrm{MAX}}} R_{i}(E) S(E, t) d E \\
& +\varepsilon_{i}(t) .
\end{aligned}
$$

This requirement (called a collocation assumption $[52,54,75,113])$ makes $S_{\text {unfold }}$ account not only for the xray data of the unknown source $S$ but also for the perturbations $\varepsilon_{i}$. Via Eqs. (12) and (13), Eq. (16) reduces to

$$
\sum_{j=1}^{N} R_{i j} S_{j}(t)=d_{i}(t)+\varepsilon_{i}(t)=D_{i}(t)
$$

where

$$
R_{i j}=\int_{E_{\mathrm{LO}}}^{E_{\mathrm{HI}}} R_{i}(E) B_{j}(E) d E .
$$

Equation (17) is equivalent to the matrix equation

$$
\mathbb{R} \mathbf{S}(t) \equiv \mathbf{D}(t),
$$

where $\mathbb{R}$ is the $N \times N$ response matrix of elements $R_{i j}$ [Eq. (18)], and $\mathbf{S}(t)$ and $\mathbf{D}(t)$ are time-dependent, $N$-dimensional vectors with the components, $S_{j}(t)$ and $D_{i}(t)$, respectively. We refer to the $S_{j}$ 's as unfold coefficients.

Several points are important here. First, it has not yet been demonstrated that Eq. (19) is, in fact, invertible or that 
the unfolded spectrum is numerically stable or physically sensible. Second, there are two energy intervals used in Eqs. (16)-(19): $[\Delta E]=\left[E_{\mathrm{LO}}, E_{\mathrm{HI}}\right]$, which defines the $R_{i j}$ 's and outside of which $S_{\text {unfold }}(E, t)$ is assumed zero; and [0, $\left.E_{\mathrm{MAX}}\right]$ over which the $R_{i}(E)$ 's and $S(E, t)$ may be nonzero. Lastly, Eq. (16) can be more generally treated as a weighted, regularized least-squares problem with further constraints ([14,49,51-53,61,79,82,83], Sec. IVA).

\section{F. Inversion of the reformulated unfold problem}

Equation (19) can be inverted if matrix $\mathbb{R}$ is nonsingular [Eq. (18)]. The vector $\mathbf{S}$ of unfold coefficients is then solved as

$$
\mathbf{S}(t)=\mathbb{R}^{-1} \mathbf{D}(t),
$$

or componentwise as

$$
S_{j}(t)=\sum_{i=1}^{N}\left(\mathbb{R}^{-1}\right)_{j i} D_{i}(t) .
$$

The spectral reconstruction then becomes

$$
S_{\text {unfold }}(E, t)=\sum_{j=1}^{N}\left[\sum_{i=1}^{N}\left(\mathbb{R}^{-1}\right)_{j i} D_{i}(t)\right] B_{j}(E),
$$

which we henceforth call the unfold or histogram unfold algorithm, unless otherwise noted. If $\mathbb{R}$ is nonsingular, $S_{\text {unfold }}(E, t)$ in this form exists for arbitrary data and is unique.

The unfold x-ray flux $\mathcal{F}_{\text {unfold }}(t)$ is then directly estimated from channel data $D_{i}(t)$. Substitution of Eq. (22) into Eq. (15) yields two equivalent expressions:

$$
\begin{aligned}
\mathcal{F}_{\text {unfold }}(t) & =\sum_{j=1}^{N} \sum_{i=1}^{N}\left[\left(\mathbb{R}^{-1}\right)_{j i} D_{i}(t)\right] \Delta E_{j} \\
& =\sum_{i=1}^{N} \sum_{j=1}^{N}\left[\Delta E_{j}\left(\mathbb{R}^{-1}\right)_{j i}\right] D_{i}(t) .
\end{aligned}
$$

This algorithm turns, however, on the condition of matrix $\mathbb{R}$. Using the basis functions $B_{j}(E)$ [Eq. (13)] and the responses of Fig. 1 in Eq. (18), one computes

$$
\mathbb{R}=\left(389 \frac{\mu \mathrm{AeV}}{\mathrm{W}}\right) \times\left(\begin{array}{ccccc}
1.0 & 0.0 & 1.8 & 3.3 & 3.0 \\
0.0 & 2.0 & 0.0 & 0.5 & 1.1 \\
0.0 & 0.1 & 3.4 & 0.2 & 0.6 \\
0.0 & 0.0 & 2.0 & 3.1 & 2.6 \\
0.0 & 0.0 & 0.0 & 0.4 & 1.2
\end{array}\right)
$$

for the $Z$ diagnostic (and similarly for response sets $\left\{R_{i a}\right\}$ and $\left\{R_{i b}\right\}$ in Fig. 2). The condition of $\mathbb{R}$ is appraised like $\mathbb{G}$ [Eq. (11)], and Fig. 6 includes the singular-value ratios, $r_{k} / r_{1}$, which indicate no ill conditioning or singularity [82]. The cond $(\mathbb{R})$ is $\sim 11$, i.e., $\sim 10$ times smaller than $\operatorname{cond}(\mathbb{G})$ and due to the independence of the $R_{i}$ 's and our choice of basis functions $B_{j}[49,50,81,82,85,122]$. SVD factoring of $\mathbb{R}$ yields $\mathbb{R}^{-1}$ :

$$
\begin{aligned}
\mathbb{R}^{-1}= & \left(2571 \frac{\mathrm{W}}{\mathrm{A}} \frac{1}{\mathrm{eV}}\right) \\
& \times\left(\begin{array}{ccccc}
1.0 & 0.0 & 0.1 & -1.0 & -0.3 \\
0.0 & 0.5 & 0.0 & 0.0 & -0.4 \\
0.0 & 0.0 & 0.3 & 0.0 & -0.2 \\
0.0 & 0.0 & -0.2 & 0.4 & -0.8 \\
0.0 & 0.0 & 0.1 & -0.1 & 1.1
\end{array}\right)
\end{aligned}
$$

\section{G. Upper-bound estimates of unfold uncertainty}

Part 2 of this article details the sensitivity of the spectral unfold $\mathbf{S}$ to data and response-function uncertainties, $\Delta \mathbf{D}$ and $\Delta \mathbb{R}$, respectively. But, an upper-bound measure to the propagated perturbation $\Delta \mathbf{S}$ can be estimated from the cond $(\mathbb{R})$. For example, for perturbations $\Delta \mathbf{D}$, one can show generally $[49,62,75,96,97,123]$ that $\|\Delta \mathbf{S}\| /\|\mathbf{S}\| \leq$ $\operatorname{cond}(\mathbb{R})\|\Delta \mathbf{D}\| /\|\mathbf{D}\|$, where $\|\mathbf{D}\|=\left(\sum_{i=1}^{N} D_{i}^{2}\right)^{1 / 2}$. [A similar relation connects $\Delta \mathbf{S}$ with cond $(\mathbb{R})$ and $\Delta \mathbb{R}$.] Now, for the $Z$ diagnostic, $\operatorname{cond}(\mathbb{R}) \simeq 11$. Hence, when only roundoff errors apply (Appendix D), $\|\Delta \mathbf{S}\| /\|\mathbf{S}\|$ is negligible, and $S_{\text {unfold }}$ is essentially noise free $\left(\varepsilon_{i}=0\right.$, as in Sec. IV below). However, should perturbations, $\Delta \mathbf{D}$ or $\Delta \mathbb{R}$, rise to the $10 \%$ (or more) level, cond $(\mathbb{R})$ could significantly distort $S_{\text {unfold }}$. This issue is rejoined in Pt. 2: Sec. II.

\section{H. Physical interpretation of the unfold coefficients $S_{j}$ for well-behaved spectral source functions}

Equations (20)-(23) comprise the entire algorithm for reconstructing the $\mathrm{x}$-ray spectrum and $\mathrm{x}$-ray flux for the $Z$ diagnostic. It is, lastly, worth checking to see if this algorithm is consistent with the well-behaved spectra assumed in Table I. This examination clarifies how the obtained unfold coefficients $S_{j}$ relate to $S(E)$.

We assume that the data in Eq. (1) are noise free, that $S(E)$ is negligible outside $[\Delta E]$, and that $S(E)$ and the $R_{i}(E)$ 's can be expanded within each bin $\left[\Delta E_{j}\right]$ about its midpoint $\bar{E}_{j}$. The integral on the right-hand side of Eq. (1) can be separated into $N$ terms [49], each integrated over the expansions of $R_{i}(E)$ and $S(E) . d_{i}$ (Appendix E) may then be expressed in terms of $\Delta E_{j}$ and midpoint values of $R_{i}, S$ (and derivatives, $R_{i}^{\prime}, S^{\prime}, R_{i}^{\prime \prime}, S^{\prime \prime}$, etc.):

$$
\begin{aligned}
d_{i}= & \sum_{j=1}^{N} R_{i j}\langle S\rangle_{j}\left\{1+\frac{\left(\Delta E_{j}\right)^{2}}{12} \frac{R_{i}^{\prime} S^{\prime}}{R_{i} S}-\frac{\left(\Delta E_{j}\right)^{4}}{24^{2}}\left[\left(\frac{R_{i}^{\prime \prime}}{R_{i}}\right)^{2}\right.\right. \\
& \left.\left.+\left(\frac{S^{\prime \prime}}{S}\right)^{2}+\cdots\right]+O\left[\left(\Delta E_{j}\right)^{6}\right]\right\},
\end{aligned}
$$

where $\langle S\rangle_{j}=\left(\Delta E_{j}\right)^{-1} \int_{E_{j}}^{E_{j+1}} S(E) d E \quad$ and $\quad R_{i j}=\int_{E_{j}}^{E_{j+1}}$ $R_{i}(E) d E \quad$ [cf. Eq. (18)]. The expression in square brackets includes unlisted cross-product terms. If $\mathbb{R}^{-1}$ exists, Eq. (26) can be substituted in the unfold algorithm [Eq. (20) or (21)] and yields $S_{j}=\langle S\rangle_{j}$ in zeroth order. 
The first correction mixes in relative slopes, and the second includes binwise peaking of $R_{i}$ and $S$. These are all terms that the a priori assumptions in Table I [124] are intended to suppress. We thus infer that $S_{j} \cong\langle S\rangle_{j}$ for generally wellbehaved spectra but that serious disagreement may occur otherwise. Also, for $S$ well behaved, $\mathcal{F}_{\text {unfold }}=$ $\sum_{j=1}^{N} S_{j} \Delta E_{j} \cong \sum_{j=1}^{N}\langle S\rangle_{j} \Delta E_{j}=\mathcal{F}_{[\Delta E]}$, which is the goal of the $Z$ diagnostic. Both conjectures are tested in Sec. IV and explained in Pt. 2: Sec. III.

\section{RESULTS: INTERNAL CONSISTENCY TESTS WITH NOISE-FREE SIMULATIONS}

The unfold algorithm [Eqs. (20)-(25)] was quantitatively tested by first simulating [51] noise-free $[\boldsymbol{\varepsilon} \approx \mathbf{0}]$ data, $\mathbf{D}(t)=\mathbf{d}(t)=\left[\int_{0}^{E_{\mathrm{MAX}}} R_{1}(E) S(E, t) d E, \ldots, \int_{0}^{E_{\mathrm{MAX}}} R_{N}(E)\right.$ $S(E, t) d E]$, from prescribed x-ray spectra $S(E, t)$ and the responses (Figs. 1 and 2), and then unfolding $\mathbf{D}(t)$. The reconstruction, $S_{\text {unfold }}(E, t)$, was then compared with the histogram average, $S_{\mathrm{AVE}}(E, t)$, of $S(E, t)$ over the same bins $\left[\Delta E_{j}\right]$ (cf. Sec. III H). Thus,

$$
\begin{aligned}
S_{\text {unfold }}(E, t) \equiv & \sum_{j=1}^{N} S_{j} B_{j}(E) \\
\equiv & \sum_{j=1}^{N}\left[\sum_{i=1}^{N}\left(\mathbb{R}^{-1}\right)_{j i}\right. \\
& \left.\times \int_{0}^{E_{\mathrm{MAX}}} R_{i}\left(E^{\prime}\right) S\left(E^{\prime}, t\right) d E^{\prime}\right] B_{j}(E),
\end{aligned}
$$

and

$$
\begin{aligned}
S_{\mathrm{AVE}}(E, t) & \equiv \sum_{j=1}^{N}\langle S\rangle_{j} B_{j}(E) \\
& \equiv \sum_{j=1}^{N}\left[\left(\Delta E_{j}\right)^{-1} \int_{E_{j}}^{E_{j+1}} S\left(E^{\prime}, t\right) d E^{\prime}\right] B_{j}(E),
\end{aligned}
$$

where $t$ is indicated for time-varying simulations. For these comparisons (a) no arbitrary normalizations were employed; (b) the same response functions were used for simulation and unfolding; (c) on physical grounds, Planckian spectra $S_{b b}(E, T)$ were primarily (but not exclusively) chosen for $S(E, t)$. Corresponding flux integrals were calculated: $\mathcal{F}_{\text {unfold }}(t)=\int_{E_{\mathrm{LO}}}^{E_{\mathrm{HI}}} S_{\text {unfold }}(E, t) d E$, $\mathcal{F}_{[\Delta E]}=\int_{E_{\mathrm{LO}}}^{E_{\mathrm{HI}}} S_{\mathrm{AVE}}(E, t) d E, \quad$ and $\quad \mathcal{F}(t)=\mathcal{F}_{[\Delta E]}+$ $\int_{0}^{E_{\mathrm{LO}}} S(E, t) d E+\int_{E_{\mathrm{HI}}}^{E_{\mathrm{MAx}}} S(E, t) d E$ [Eqs. (15), (4), and (3)].

Following experimental interests, we defined binwise and integral figures of merit for assessing the algorithm. Thus, for example, (a) $S_{j}-\langle S\rangle_{j}$ was defined as the absolute unfold distortion; (b) $\left[S_{j}-\langle S\rangle_{j}\right] /\langle S\rangle_{j}$ as the relative unfold distortion; and (c) $S_{j} /\langle S\rangle_{j}$ as the spectral recovery. Similar measures were defined for $\mathcal{F}_{\text {unfold }}, \mathcal{F}_{[\Delta E]}$, and $\mathcal{F}$. These measures are generalized in Pt. 2: Sec. III. \{Other measures of closeness, e.g., $\Sigma_{i}\left[D_{i}-\int R_{i}(E) S_{\text {unfold }}(E) d E\right]^{2}$ and
$\int\left[S(E)-S_{\text {unfold }}(E)\right]^{2} d E$, are particularly germane to statistical and functional analyses but are not discussed here.\}

Also defined were separate characterizations of the simulating spectra $S$. One of these was a crude, binwise measure of the spectral shape, $\langle S\rangle_{j} / S_{P}$, where $S_{P}=$ $\max \{S(E)\}$ in $\left[0, E_{\mathrm{MAX}}\right]$; this ratio helped to appraise $S_{j} /\langle S\rangle_{j}$, especially when $\langle S\rangle_{j} / S_{P} \cong 0$. A second measure [121] gauged how well (in an rms sense) the chosen $B_{j}$ 's could approximate $S$.

The simulations fell into two classes. The first prescribed time-fixed spectra $S(E)$ : binwise spectral- and flux-recovery measures were examined. The second class simulated time-varying spectra $S(E, t)$ : flux recovery and temporal features (e.g., pulse widths) were studied.

\section{A. Time-independent, noise-free data}

The first simulation tested the unfold algorithm against the specific constraints of Table I. A non-Planckian $S(E)$ (Fig. 7) was chosen that was broad compared to the $R_{i}(E)$ 's, continuous, and non-negative inside the unfold domain $[\Delta E]$ — but zero outside. Figure 7 compares $S(E)$ with $S_{\mathrm{AVE}}(E)$ and $S_{\text {unfold }}(E)$. One sees that, although $S_{\text {unfold }}(E)$ cannot resolve the detailed behavior of $S(E)$, it still displays the correct qualitative shape; and the unfold coefficients $S_{j}$ compare quantitatively well with $\langle S\rangle_{j}$ over most of $[\Delta E]$. The flux measures $\mathcal{F}_{\text {unfold }}$ and $\mathcal{F}$ agree within $4 \%$. (Here, $\mathcal{F}_{[\Delta E]} \equiv \mathcal{F}$ due to the defined domain of $S$.)

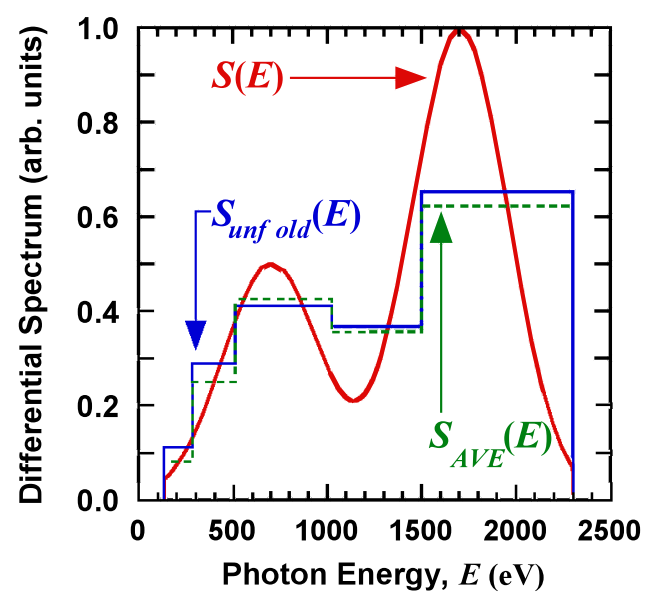

FIG. 7. Comparison of a non-Planckian, test spectrum $S(E)$ with $S_{\mathrm{AVE}}(E)$ and $S_{\text {unfold }}(E)$. Here, $S(E)$ satisfies Table I, and $S_{\mathrm{AVE}}(E)$ is constructed from $\langle S\rangle_{j} .\left[S_{\mathrm{AVE}}(E)\right.$ is the best leastsquares approximation to $S(E)$ by the given basis functions $B_{j}(E)$; its rms measure [121] of closeness is 0.43 on a $0-1$ scale, where 0 signifies exact agreement.] $S_{\text {unfold }}(E)$ is the unfold for data simulations with $S(E)$ and $\left\{R_{1}, \ldots, R_{5}\right\}$ (Fig. 1); its relative distortion, $\left(S_{j} /\langle S\rangle_{j}-1\right)$, ranges from a few percent near the spectral peak to $\sim 36 \%$ in the lowest $x$-ray energy bin, which contains relatively little flux. 
A series of ten Planckian spectra, $S_{b b}(E, 25 \mathrm{eV} \leq T \leq$ $252 \mathrm{eV}$ ), was also chosen to test the unfold algorithm. These simulating spectra largely satisfy the constraints of Table I, although $S_{b b}(E, T)$ progressively violates assumptions \#4 and \#5 with decreasing $T$. Figures 8(a)-8(d) compare $S_{b b}(E, T), S_{\mathrm{AVE}}(E, T)$, and $S_{\text {unfold }}(E, T)$ for $T=250$, 175,100 , and $25 \mathrm{eV}$. Table III lists recoveries, $S_{j} /\left\langle S_{b b}\right\rangle_{j}$, and spectral source ratios, $\left\langle S_{b b}\right\rangle_{j} /\left(S_{b b}\right)_{P}$.

The results in Fig. 8 and Table III illustrate several features of the unfold algorithm. First, within $[\Delta E]$ $S_{\text {unfold }}$ reproduces the general shape and magnitude of all the Planckian spectra considered, as above. Second, the spectral recovery $S_{j} /\left\langle S_{b b}\right\rangle_{j} \geqslant 80 \%$ for $125 \mathrm{eV} \leq T \leq$ $250 \mathrm{eV}$, except where $\langle S\rangle_{j} / S_{P} \cong 0$; spectral recovery is, however, less successful at lower spectral temperatures because $S_{b b}(E, T)$ progressively falls below the detection cutoff at $E_{\mathrm{LO}}(137 \mathrm{eV})$ and is thus less well approximated by the basis functions. (The rms closeness measures [121] are noted in the figure captions.) A third trend is the occasional appearance of unfold coefficients $S_{j}<0$, a phenomenon also correlated with bins nearly devoid of spectrum \{e.g., $S_{4}$ for $T=100 \mathrm{eV}$ [Fig. 8(c)], where $\left.\left\langle S_{b b}\right\rangle_{4} /\left(S_{b b}\right)_{P} \cong 0.01\right\}$. Such behavior has been noted elsewhere [125] for a different diagnostic and can be understood for arbitrary spectral functions by the methods of Pt. 2: Sec. III. Thus, in these noise-free tests, the $Z$ diagnostic and its unfold algorithm perform reasonably well within the designed $x$-ray regime.

The x-ray flux estimate $\mathcal{F}_{\text {unfold }}$ was compared with both the total flux $\mathcal{F}$ and the flux $\mathcal{F}_{[\Delta E]}$ of each simulating $S_{b b}(E, T)$. Figure 9(a) plots $\mathcal{F}(T)$, $\mathcal{F}_{[\Delta E]}(T)$, and $\mathcal{F}_{\text {unfold }}(T)$ versus $T$. The solid blue dots denote $\mathcal{F}$, for which $T^{4}$ scaling is verified (dashed curve) $[5,7,37,98]$. The solid red squares plot $\mathcal{F}_{[\Delta E]}$, for which $\mathrm{x}$ rays of energy outside $[\Delta E]$ are not counted [Eq. (4)]. Lastly, the solid green dots give $\mathcal{F}_{\text {unfold }}$ [Eq. (23)]. Figure 9(a) shows fair agreement between $\mathcal{F}_{[\Delta E]}$ and $\mathcal{F}_{\text {unfold }}$ at low spectral temperatures, where $\left(\mathcal{F}_{[\Delta E]}-\mathcal{F}_{\text {unfold }}\right)$ is attributable to a lack of approximation of $S_{b b}(E, T)$ by the $B_{j}(E)$ 's [cf. Fig. 8(d)]; the larger discrepancies, $\left(\mathcal{F}-\mathcal{F}_{[\Delta E]}\right)$ and $\left(\mathcal{F}-\mathcal{F}_{\text {unfold }}\right)$, for the same temperatures are principally due to lowenergy x rays counted in $\mathcal{F}$, but not in $\mathcal{F}_{[\Delta E]}$ or $\mathcal{F}_{\text {unfold }}$ [cf. Table IV]. With increasing $T$, however, agreement
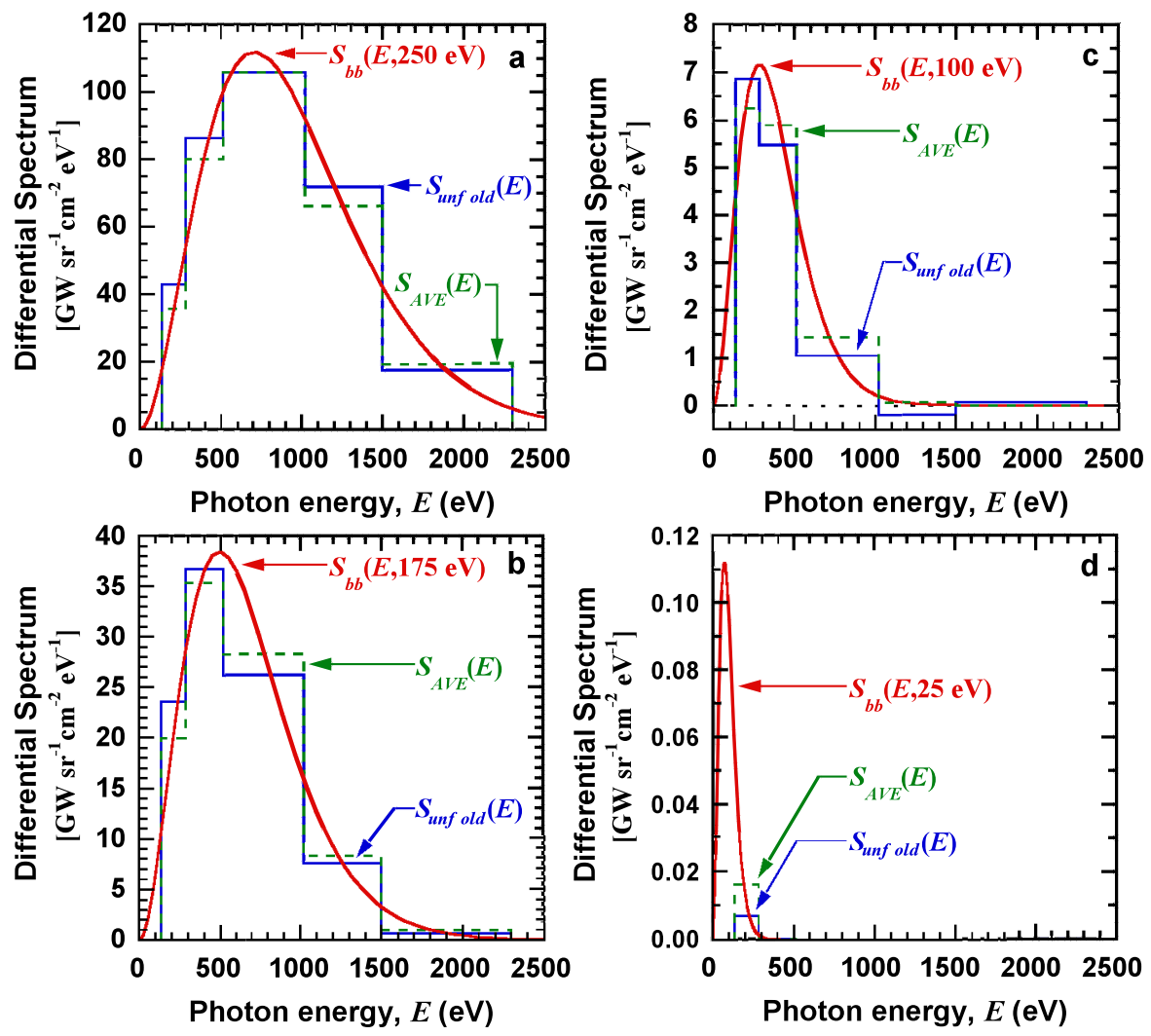

FIG. 8. Comparison of four Planckian test spectra $S_{b b}(E, T)$ of noted spectral temperatures, bin-averaged spectra $S_{\mathrm{AVE}}(E)$, and unfolded spectra $S_{\text {unfold }}(E)$. Here, $S_{\mathrm{AVE}}(E)$ reasonably approximates $S_{b b}$ for $T=250,175$, and $100 \mathrm{eV}$ (rms closeness measures 0.18 , $0.23,0.33$, respectively) but poorly approximates $S_{b b}(E, 25 \mathrm{eV})$ [rms closeness $\sim 0.98$ ]. Unfolded spectra $S_{\text {unfold }}(E)$ from corresponding data simulations show general binwise agreement with $S_{\mathrm{AVE}}(E)$ down to $T=100 \mathrm{eV}(\mathrm{c})$, where a negative unfold value is obtained in bin $\left[\Delta E_{4}\right]$. There is also significant disagreement between $S_{\mathrm{AVE}}(E)$ and $S_{\text {unfold }}(E)$ for $S_{b b}(E, 25 \mathrm{eV})$ (cf. Table III). 
TABLE III. Ratio of the unfold coefficient to the average of simulating Planckian spectrum $S_{j} /\langle S\rangle_{j}$ in each unfold bin $j$ for various simulating blackbody spectra, parametrized by temperature $T$ in eV; upper entries highlighted in bold font show the bins in which $S_{j}$ agrees with $\langle S\rangle_{j}$ to within $\pm 20 \%$. (The spectral fractional distortion, cited in the text, is $S_{j} /\langle S\rangle_{j}-1$.) Also shown are the bin intervals $E_{j}$ to $E_{j+1}$ and widths $\Delta E_{j}$ in $\mathrm{eV}$. The ratio $\langle S\rangle_{j} / S_{P}$ of the binwise average to the peak of the simulating spectrum is given in parentheses at each temperature and bin to indicate the approximate shape of $S_{b b}$ in $[\Delta E]$.

\begin{tabular}{lccccc}
\hline \hline Ratio $\rightarrow$ & $S_{1} /\langle S\rangle_{1}\left(\langle S\rangle_{1} / S_{p}\right)$ & $S_{2} /\langle S\rangle_{2}\left(\langle S\rangle_{2} / S_{p}\right)$ & $S_{3} /\langle S\rangle_{3}\left(\langle S\rangle_{3} / S_{p}\right)$ & $S_{4} /\langle S\rangle_{4}\left(\langle S\rangle_{4} / S_{p}\right)$ & $S_{5} /\langle S\rangle_{5}\left(\langle S\rangle_{5} / S_{\mathrm{p}}\right)$ \\
$\operatorname{Bin}\left[\Delta E_{j}\right] \rightarrow T(\mathrm{eV}) \downarrow$ & $137-284 \mathrm{eV}$ & $284-513 \mathrm{eV}$ & $513-1020 \mathrm{eV}$ & $1020-1500 \mathrm{eV}$ & $1500-2300 \mathrm{eV}$ \\
\hline 25 & 0.43 & 0.34 & -22 & $10^{9}$ & $10^{38}$ \\
& $(0.14)$ & $(0.00)$ & $(0.00)$ & $(0.00)$ & $(0.00)$ \\
50 & $\mathbf{0 . 8 4}$ & 0.67 & 0.19 & -70 & $10^{38}$ \\
& $(0.77)$ & $(0.16)$ & $(0.00)$ & $(0.00)$ & $(0.00)$ \\
75 & $\mathbf{1 . 0 1}$ & $\mathbf{0 . 8 3}$ & 0.58 & -30 & $10^{38}$ \\
& $(0.95)$ & $(0.54)$ & $(0.06)$ & $(0.00)$ & $(0.00)$ \\
100 & $\mathbf{1 . 1 0}$ & $\mathbf{0 . 9 3}$ & 0.73 & -3.5 & 81 \\
& $(0.87)$ & $(0.82)$ & $(0.20)$ & $(0.01)$ & $(0.00)$ \\
125 & $\mathbf{1 . 1 4}$ & $\mathbf{0 . 9 8}$ & $\mathbf{0 . 8 2}$ & -0.2 & 6.9 \\
& $(0.75)$ & $(0.95)$ & $(0.39)$ & $(0.04)$ & $(0.00)$ \\
150 & $\mathbf{1 . 1 7}$ & $\mathbf{1 . 0 2}$ & $\mathbf{0 . 8 8}$ & 0.63 & 1.24 \\
& $(0.62)$ & $(0.96)$ & $(0.58)$ & $(0.11)$ & $(0.01)$ \\
175 & $\mathbf{1 . 1 8}$ & $\mathbf{1 . 0 4}$ & $\mathbf{0 . 9 3}$ & $\mathbf{0 . 9 1}$ & 0.66 \\
& $(0.52)$ & $(0.92)$ & $(0.74)$ & $(0.21)$ & $(0.03)$ \\
200 & $\mathbf{1 . 1 9}$ & $\mathbf{1 . 0 6}$ & $\mathbf{0 . 9 6}$ & $\mathbf{1 . 0 2}$ & 0.69 \\
& $(0.44)$ & $(0.86)$ & $(0.84)$ & $(0.34)$ & $(0.06)$ \\
225 & $\mathbf{0 . 8 4}$ & $\mathbf{0 . 9 4}$ & $\mathbf{1 . 0 2}$ & $\mathbf{0 . 9 4}$ & 1.26 \\
& $(0.37)$ & $(0.78)$ & $(0.91)$ & $(0.47)$ & $(0.11)$ \\
250 & $\mathbf{1 . 2 0}$ & $\mathbf{1 . 0 8}$ & $\mathbf{1 . 0 0}$ & $\mathbf{1 . 0 9}$ & $\mathbf{0 . 8 9}$ \\
& $(0.32)$ & $(0.72)$ & $(0.95)$ & $(0.59)$ & $(0.18)$ \\
\hline \hline
\end{tabular}

among the three flux measures improves as $S_{b b}(E, T)$ expands across the response functions (Fig. 1).

The flux-recovery ratios, $\mathcal{F}_{\text {unfold }}(T) / \mathcal{F}_{[\Delta E]}(T)$ and $\mathcal{F}_{\text {unfold }}(T) / \mathcal{F}(T)$, are plotted in Fig. 9(b). On this scale, one sees that $\mathcal{F}_{\text {unfold }}$ recovers at least $90 \%$ of $\mathcal{F}_{[\Delta E]}$ for $T \geq 100 \mathrm{eV}$. In fact, for $200 \mathrm{eV} \leq T \leq 250 \mathrm{eV}$, the recovery slightly exceeds $100 \%$ because high-energy x rays spilling over the upper cutoff $E_{\mathrm{HI}}(2300 \mathrm{eV})$ are still detected by the response functions [cf. Eq. (27), Figs. 2 and 8 and Pt. 2: Sec. IIIC]. The sharp falloff in recovery for $T<100 \mathrm{eV}$ corresponds to the spectral disagreements noted in Fig. 9(a). Together these curves suggest that the most realistic goal for the $Z$ diagnostic (as designed) is to recover $\mathcal{F}_{[\Delta E]}$, rather than $\mathcal{F}$-unless lower-energy channel responses are included.

These tests also raise two other important issues: (a) How representative of $S(E)$ are unfolds obtained from different sets of response functions? And, (b) how does one deal with negative unfold coefficients $S_{j}$ ? To address the first point, separate data sets were simulated from $S_{b b}(E, 200 \mathrm{eV})$ and two response-function sets, $R_{1}, \ldots, R_{5}$ and $R_{1 a}, \ldots, R_{5 a}$ (Fig. 2). Reconstructed spectra unfolded with the same responses that simulated each data set (e.g., via $\mathbb{R}^{-1}$ for $R_{1}, \ldots, R_{5}$, and $\mathbb{R}_{a}^{-1}$ for $R_{1 a}, \ldots, R_{5 a}$ ) were called consistent unfolds. In Fig. 10(a) these unfolded spectra are nearly indistinguishable, both in agreement with $S_{b b}(E, 200 \mathrm{eV})$ (cf. Table III). Hence, separately calibrated diagnostics viewing the same spectrum are expected to agree at least in first order [126]. Figure 10(a) also shows the result of unfolding inconsistently: i.e., data simulated from $R_{1 a}, \ldots, R_{5 a}$ were unfolded with $\mathbb{R}^{-1}$ (instead of $\mathbb{R}_{a}^{-1}$ ). This case is examined further in Pt. 2: Sec. II B.

The second issue concerns unfold coefficients, $S_{j}<0$, which occasionally appeared in Planckian test simulations (Table III, Fig. 8) and against which we have not explicitly protected the unfold algorithm (Sec. IIIE). In fact, the effect of a non-negativity constraint, $S_{j+} \geq 0$, was tested with the method of Lawson and Hanson [83], which iteratively inverts Eq. (19) as a least-squares problem and is incorporated in the UFO unfold code [127]. The spectrum $S_{b b}(E, 100 \mathrm{eV})$ was selected for this test because it produced a pronounced negative unfold component in Fig. 8(c). Figure 10 (b) compares unfolds with $\left(S_{\text {unfold }+}\right)$ and without ( $\left.S_{\text {unfold }}\right)$ this constraint, and it is clear that the additional constraint merely zeros out the troublesome negative value of $S_{4}$ in $S_{\text {unfold }}$, while readjusting the other $S_{j+}$ 's. More importantly, $\mathcal{F}_{\text {unfold+ }}$ (with the constraint) disagrees with $\mathcal{F}_{\text {unfold }}$ by no more than $\sim 2 \%$. In our experience, such unfold behavior is typical for such spectra, and we have thus favored the simplicity of an exact solution for the $Z$ diagnostic over a non-negativity constraint (cf. Pt. 2: Secs. III B and III C). 


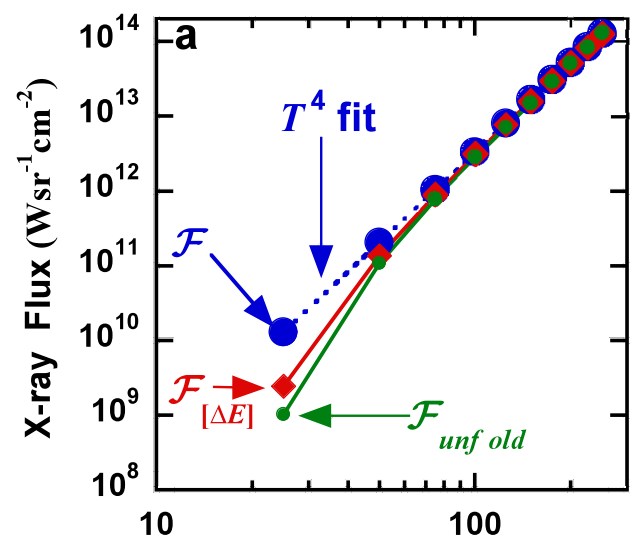

Spectral Temperature, $T(\mathrm{eV})$

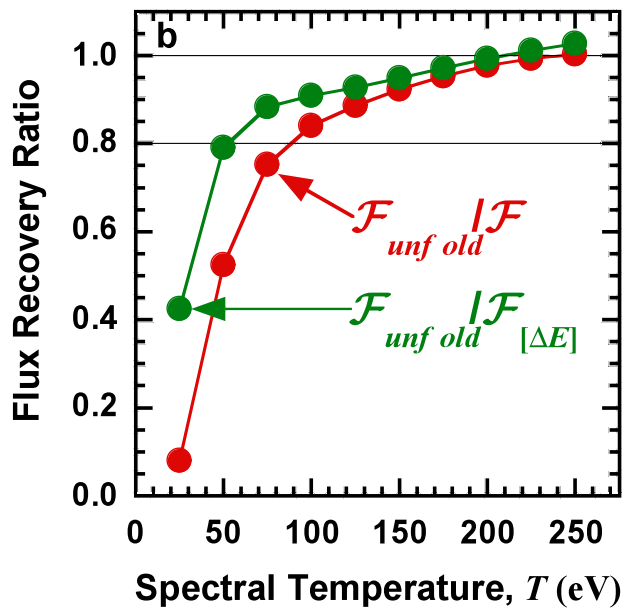

FIG. 9. Comparison of x-ray flux measures, $\mathcal{F}(T), \mathcal{F}_{[\Delta E]}(T)$, and $\mathcal{F}_{\text {unfold }}(T)$, for the Planckian test spectra $S_{b b}(E, 25 \mathrm{eV} \leq T \leq$ $250 \mathrm{eV}$ ) above. Part (a) shows each measure on an absolute scale. The dashed straight line associated with $\mathcal{F}(T)$ is a least-squares, power-law fit that verifies $T^{4}$ scaling. (Other broken lines are meant to guide the eye.) Part (b) shows flux-recovery ratios, $\mathcal{F}_{\text {unfold }}(T) / \mathcal{F}_{[\Delta E]}(T)$ and $\mathcal{F}_{\text {unfold }}(T) / \mathcal{F}(T)$. For $T \geq 100 \mathrm{eV}$, $\mathcal{F}_{\text {unfold }}(T)$ recovers $\mathcal{F}_{[\Delta E]}$ and $\mathcal{F}$ within $20 \%$ (cf. Table IV).

TABLE IV. Ratio of unfolded flux $\mathcal{F}_{\text {unfold }}$ to the two measures, $\mathcal{F}$ and $\mathcal{F}_{[\Delta E]}$ of incident flux in a series of blackbody spectra, parametrized by temperature $T$. The simulations use response functions $R_{1}, \ldots, R_{5}$ (Fig. 1). Values $S_{j}<0$ are included in $\mathcal{F}_{\text {unfold }}$ (cf. Table III and Sec. VI).

\begin{tabular}{lccc}
\hline \hline$T(\mathrm{eV})$ & $\mathcal{F}_{\text {unfold }} / \mathcal{F}$ & $\mathcal{F}_{[\Delta E]} / \mathcal{F}$ & $\mathcal{F}_{\text {unfold }} / \mathcal{F}_{[\Delta E]}$ \\
\hline 25 & 0.08 & 0.19 & 0.42 \\
50 & 0.52 & 0.66 & 0.79 \\
75 & 0.75 & 0.85 & 0.88 \\
100 & 0.84 & 0.92 & 0.91 \\
125 & 0.89 & 0.96 & 0.93 \\
150 & 0.92 & 0.97 & 0.95 \\
175 & 0.95 & 0.98 & 0.97 \\
200 & 0.98 & 0.99 & 0.99 \\
225 & 0.99 & 0.98 & 1.01 \\
250 & 1.00 & 0.97 & 1.03 \\
\hline \hline
\end{tabular}
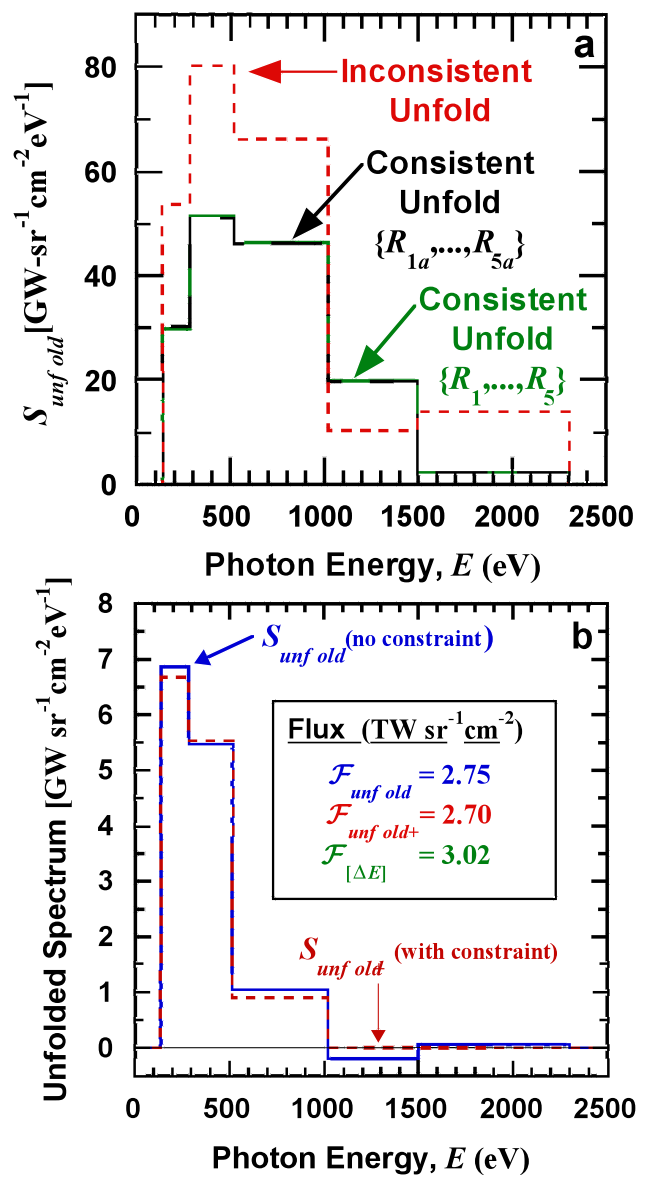

FIG. 10. Dependence of $S_{\text {unfold }}$ on the response functions and on a non-negativity constraint. In Fig. 10(a), the solid (green) and dashed (black) curves represent reconstructed spectra that have been consistently simulated and unfolded. The dotted (red) histogram was obtained when data are simulated and unfolded inconsistently. Figure 10(b) shows the effect of applying a nonnegativity constraint to the simulation and unfold of $S_{b b}(E, 100 \mathrm{eV})$ in Fig. 8(c).

\section{B. Time-dependent, noise-free data}

The temperature dependence of $\mathcal{F}_{\text {unfold }} / \mathcal{F}_{[\Delta E]}$ [Fig. 9(b) and Table IV] implies that the flux recovery $\mathcal{F}_{\text {unfold }} / \mathcal{F}_{[\Delta E]}$ varies with time for pulsed spectra $S(E, t)$ and thus, in principle, affects the power-pulse width and x-ray fluences. To quantify this connection, we again focus on Planckian sources $S_{b b}(E, T)$, now with prescribed time dependences $T(t)$ in temperature.

For this analysis, two temperature histories, $T_{150}(t)$ and $T_{225}(t)$, were synthesized from an experimental XRD signal. These histories had the following form:

$$
\left.\begin{array}{l}
T_{150}(t)=(150 \mathrm{eV}) \\
T_{225}(t)=(225 \mathrm{eV})
\end{array}\right\} \times \Omega(t)
$$

where $\Omega(t)$ is an arbitrary, peak-normalized function of time [128]. $T_{150}(t)$ and $T_{225}(t)$ are plotted in Fig. 11(a) 
. The power range, $\left(T_{\max } / T_{\min }\right)^{4}$, for these histories is $\sim 50$ and 200, respectively.

Data simulations for $S_{b b}\left[E, T_{150}(t)\right]$ and $S_{b b}\left[E, T_{225}(t)\right]$, coupled to responses, $R_{1 a}, \ldots, R_{5 a}$ (Fig. 2), were obtained as above [Eq. (22)], and the resulting time-dependent data were unfolded consistently with the inverse matrix $\mathbb{R}_{a}^{-1}$. Flux estimates, $\mathcal{F}_{[\Delta E]}(t)$ and $\mathcal{F}_{\text {unfold }}(t)$, were computed from Eqs. (4) and (23), respectively.

Figure 11(b) shows the source flux, $\mathcal{F}_{[\Delta E]}(t)$, and the flux-recovery ratio, $\mathcal{F}_{\text {unfold }}(t) / \mathcal{F}_{[\Delta E]}(t)$, for $T_{150}(t)$ and $T_{225}(t)$ in these simulations. Several points are of interest. (a) As expected from Sec. IVA, the flux recovery obtained from these responses agrees well with Fig. 9(b), based on $R_{1}, \ldots, R_{5}$ (Fig. 2). (b) Because $\mathcal{F}_{\text {unfold }}(t) / \mathcal{F}_{[\Delta E]}(t)$ is not
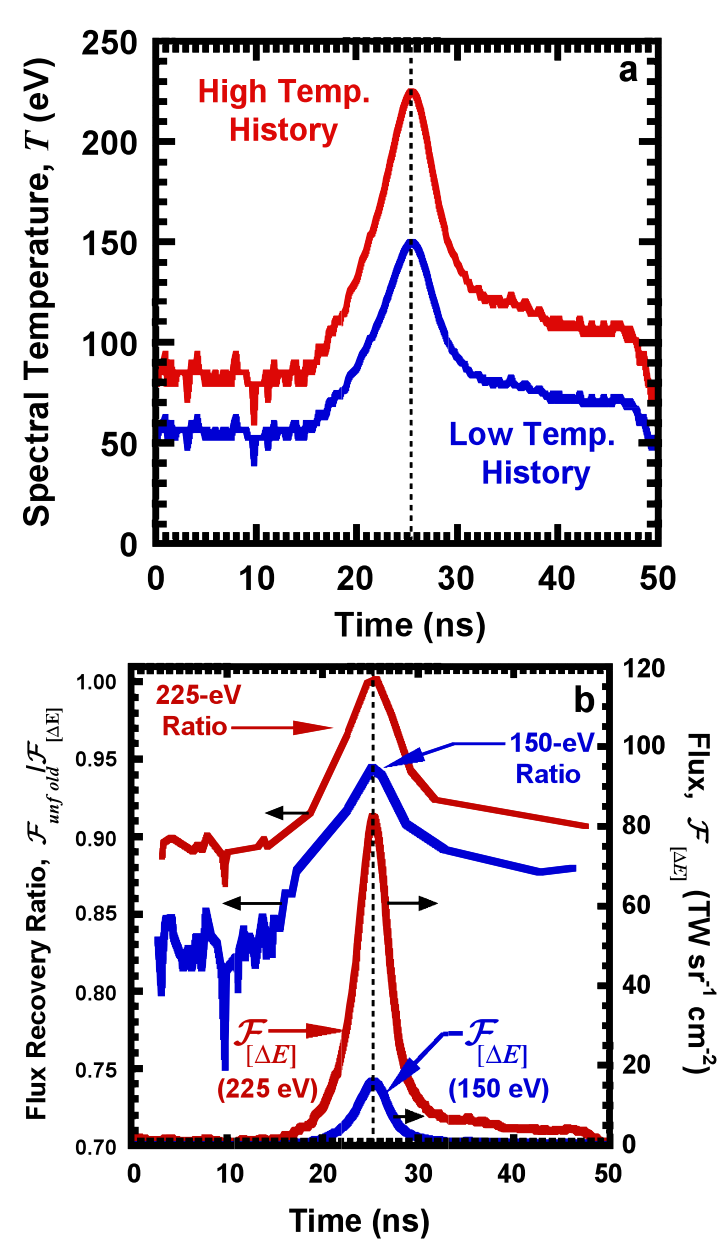

FIG. 11. Time-dependent simulations. Part (a) shows two prescribed temperature histories, $T_{150}(t)$ and $T_{225}(t)$, with peak values of 150 and $225 \mathrm{eV}$. Time-dependent Planckian spectra, $S_{b b}\left[E, T_{150}(t)\right]$ and $S_{b b}\left[E, T_{225}(t)\right]$, were generated from these profiles; and, for each, the flux $\mathcal{F}_{[\Delta E]}(t)$ in the unfold interval $[\Delta E]$ was calculated. Time-varying channel data were also simulated and unfolded to produce a corresponding unfold flux estimate $\mathcal{F}_{\text {unfold }}(t)$. In part (b) the lower curves (right scale) depict $\mathcal{F}_{[\Delta E]}(t)$ for the two histories; the upper curves (left scale) are the corresponding unfold flux-recovery ratios, $\mathcal{F}_{\text {unfold }}(t) / \mathcal{F}_{[\Delta E]}(t)$. constant, the temporal shapes of $\mathcal{F}_{[\Delta E]}(t)$ and $\mathcal{F}_{\text {unfold }}(t)$ differ slightly: $\mathcal{F}_{\text {unfold }}(t)$ is narrower (FWHM) by $\sim 2 \%-6 \%$ [for $T_{150}(t)$ and $T_{225}(t)$, respectively].

\section{DISCUSSION: COMPARISONS WITH INDEPENDENT ALGORITHMS AND DIAGNOSTICS}

Thus far, the utility of the flux estimate $\mathcal{F}_{\text {unfold }}$ rests on self-consistency tests with prescribed spectra. But, further verification of this inversion technique requires independent testing. Here, we compare the histogram results (a) with an x-ray-flux estimate derived without unfolding and applied to experimental $z$-pinch data; (b) with unfolded $z$-pinch spectra obtained by the code, SHAMPC; and (c) with laser-produced spectra unfolded by the UNSPEC code using DANTE data.

We have previously reported a method of estimating $\mathrm{x}$-ray flux from filtered-XRD-channel data that does not involve spectral unfolding [29]. This estimate, denoted $\mathcal{F}_{L S}$, results from a numerical "equalization" of the response functions:

$$
\mathcal{F}_{L S} \equiv \int_{0}^{E_{\mathrm{MAX}}}\left[\sum_{i=1}^{N} a_{i} R_{i}(E)\right] S(E) d E \equiv \sum_{i=1}^{N} a_{i} d_{i} .
$$

The coefficients $a_{i}$ are chosen to minimize the function, $\int\left[\sum_{i=1}^{N} a_{i} R_{i}(E)-1\right]^{2} d E$ over $[\Delta E]$, subject to the constraint $\left\langle\sum_{i=1}^{N} a_{i} R_{i}\right\rangle=1$. Coefficients for responses $R_{1}, \ldots, R_{5}$ (Fig. 1) obtained in this constrained-leastsquares (LS) procedure are listed in Table V. For comparison, the unfold flux estimate $\mathcal{F}_{\text {unfold }}$ [Eq. (23)] may also be cast into this form by defining corresponding coefficients $\left(a_{\text {unfold }}\right)_{i}$ as follows:

$$
\left(a_{\text {unfold }}\right)_{i}=\sum_{j=1}^{N}\left(\Delta E_{j}\right)\left(\mathbb{R}^{-1}\right)_{j i},
$$

which are also listed in Table $\mathrm{V}$ for the same response functions (Fig. 1) and $[\Delta E]$. These two sets of coefficients reveal striking similarities in sign, magnitude, and channel dependence, although $\left|\left(a_{\text {unfold }}\right)_{i}\right|$ systematically exceeds $\left|a_{i}\right|$, except for $i=5$. For Planckian spectra $(100 \mathrm{eV} \leq$ $T \leq 250 \mathrm{eV}), \mathcal{F}_{\text {unfold }} / \mathcal{F}_{[\Delta E]}$ exceeds $\mathcal{F}_{L S} / \mathcal{F}_{[\Delta E]}$ but the

TABLE V. Comparison of coefficients for flux passband functions, $\sum_{i} a_{i} R_{i}(E)$ and $\sum_{i}\left(a_{\text {unfold }}\right)_{i} R_{i}(E)$, calculated by the LS method (Ref. [29]) and the unfold method of this article, respectively.

\begin{tabular}{lcc}
\hline \hline Response $R_{i}$ & $a_{i}(\mu \mathrm{A} / \mathrm{W})$ & $\left(a_{\text {unfold }}\right)_{i}(\mu \mathrm{A} / \mathrm{W})$ \\
\hline 1 & 0.28 & 0.38 \\
2 & 0.26 & 0.28 \\
3 & 0.25 & 0.26 \\
4 & -0.06 & -0.14 \\
5 & 0.95 & 0.71 \\
\hline \hline
\end{tabular}


disagreement is $\sim 10 \%$ [Ref. 29, Fig. 3]. We take this comparison as independent support for the unfold algorithm and our definition of $\mathcal{F}_{\text {unfold }}$. Data-noise propagation is similar for $\mathcal{F}_{L S}$ and $\mathcal{F}_{\text {unfold }}$ [cf. [29] and Pt. 2: Sec. II, Fig. 1(b)].

In a second test, experimental data from the $Z$ diagnostic were analyzed independently by two different unfold techniques, thus addressing differences in the a priori assumptions (e.g., basis functions, prescribed constraints, etc.) of both algorithms. The histogram unfold $S_{\text {unfold }}(E)$ was obtained in the usual way [Eqs. (22) and (23)], and a second spectral estimate $S_{\text {shampc }}(E)$ was reconstructed by the SHAMPC code, constrained to yield a smooth, non-negative spectrum. Idzorek $[58,129]$ has reported this comparison for vacuum hohlraum experiments at the $Z$ accelerator: at peak radiated power, one finds reasonable agreement between $S_{\text {shampc }}(E)$ and $S_{\text {unfold }}(E)$, although $S_{\text {shampc }}(E)$ shows some unphysical distortions of slope at the major characteristic edges in the response functions.

A third test compares the histogram unfold method with still another unfold algorithm, both applied to an x-ray source and spectral diagnostic different from above. The source in this comparison was a gold target, onto which nine laser beams of the NOVA laser facility $[59,60]$ focused an 18-kJ, 5ns-wide, square pulse of 3- $\omega$ photons (shot \#26020710, 7 February 1996). Soft x rays (100 eV$3 \mathrm{keV}$ ) were detected by the DANTE spectrometer [46,47], a 15-channel calibrated XRD array that employs $\mathrm{x}$-ray mirrors, filters, and auxiliary background detectors. The DANTE channel data were independently unfolded by the UNSPEC $[47,130]$ code (used routinely for this diagnostic) and by the histogram unfold method presented here. [UNSPEC employs an iterative unfold technique, as opposed to the matrix inversion of Eq. (20).] For this comparison, the matrix $\mathbb{R}$ and the unfold bins $\left[\Delta E_{j}\right]$ of the histogram unfold algorithm conformed to the DANTE response functions. Moreover, since only the seven lowest-energy DANTE channels were judged to contain signal above noise in this experiment, the histogram unfold was based solely on these $N=7$ signals and the corresponding response functions; higher-energy responses and waveforms, including background channels, were ignored. No normalizations, either between the unfold methods or with respect to other measurements, were employed.

Figures 12(a) and 12(b) compare the results in this test. Figure 12(a) shows the unfolded differential spectra, $S_{\text {unspec }}\left(E, t_{p}\right)$ and $S_{\text {unfold }}\left(E, t_{p}\right)$, at peak emitted x-ray power $\left(t_{p}=4.9 \mathrm{~ns}\right)$. Both techniques obtained a spectrum that reaches a maximum between 200 and $300 \mathrm{eV}$ but that also includes a weak, non-Planckian feature near $1 \mathrm{keV}$. $S_{\text {unspec }}$ exceeded $S_{\text {unfold }}$ by $\sim 5 \%$ at the spectral maximum and by $\sim 25 \%$ in the adjacent energy bins. The corresponding peak brightness temperature, computed from these two spectra, was estimated to be $90.5 \mathrm{eV}$ by UNSPEC and $88.3 \mathrm{eV}$ by the histogram algorithm. Figure 12(b) shows

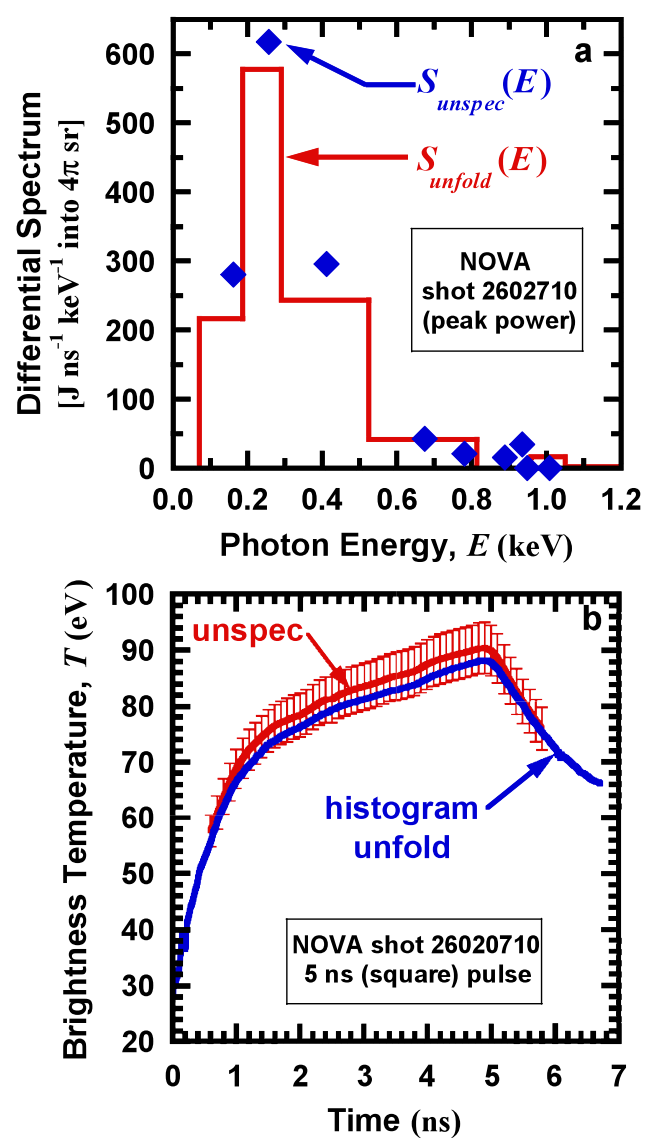

FIG. 12. The results of two unfold methods applied to DANTE data are compared. Data were taken at the NOVA facility (shot 26020710) and were independently unfolded with the UNSPEC code and the histogram unfold technique, given here. No normalizations were used. Part (a) compares the unfolded differential spectra, $S_{\text {unspec }}\left(E, t_{p}\right)$ and $S_{\text {unfold }}\left(E, t_{p}\right)$, at peak emitted x-ray power $\left(t_{p}=4.9 \mathrm{~ns}\right)$. Part (b) gives the time-dependent brightness temperature from these two methods. Vertical bars attached to the UNSPEC curve represent $\pm 5 \%$-vernier bounds to guide the eye.

that such agreement in brightness temperature extended throughout this experiment: both unfold methods yielded similar temperature histories, rising monotonically during laser heating and then falling after the laser was switched off. The 5\%-vertical bars attached to the upper (DANTE) curve indicate that the two methods disagreed in brightness temperature by no more than $\sim 3 \%$; the associated disagreement in estimated flux was, therefore, $\leq 12 \%$.

\section{SUMMARY AND CONCLUDING REMARKS}

The first part of this article formulated an unfolding algorithm to estimate $\mathrm{x}$-ray flux information from continuum x-ray spectra emitted by $z$ pinches from an $N$-channel, filtered-detector-array (Sec. I). The overall strategy was to integrate a coarse reconstruction of the spectrum. A priori constraints controlled the ill posedness of this inversion problem and yielded a unique reconstruction (Sec. II). 
These constraints were derived by studying the domain, independence, and resolution of the responses as well as expected general properties of the experimental spectra (Sec. III, Table I): a Planckian representation of the reconstruction was rejected due to a lack of channel-data fit (Sec. II D). The resulting reconstruction was a histogram, determined by an exact inversion of the data (Sec. IIIF). Algorithm-induced distortions were studied for Planckian $(25 \mathrm{eV} \leq T \leq 250 \mathrm{eV})$ and non-Planckian spectra with noise-free (simulated) data. Incident and unfolded flux values agreed to within $\sim 10 \%$ for spectral temperatures of $75 \mathrm{eV} \leq T \leq 250 \mathrm{eV}$, a result which appeared adequate for the assigned diagnostic goal with these spectra; larger distortions at lower $T$ were caused by undetected $\mathrm{x}$ rays and deteriorating spectral approximation. Some reconstructions showed minor negative behavior, which could be zeroed out with an added non-negativity constraint; but, since the overall reconstructed shape and flux were only marginally affected by this constraint [Fig. 10(b) ], non-negativity was not routinely imposed. Variant response functions were examined (Sec. IVA). Timedependent data simulations indicated slight temporal narrowing of the unfolded flux with incident $\mathrm{x}$-ray flux (Sec. IV B). Detailed comparisons were made to other published techniques and other diagnostics with experimental data (Sec. V).

The following remarks are, however, in order: (1) The first-order algorithm used here is adequate for a flux estimate from $Z$-diagnostic data because (a) the dominant experimental spectra are piecewise continuous and of approximately known energy bounds; (b) the responses are independent and together spectrally resolving for a coarse energy partition; and (c) only a similarly coarse spectral reconstruction is needed. (2) But, this approach is not a cure-all, especially for significantly different and nearly singular response-function sets. (Spectacular failures and alternate procedures have been well documented $[49,50,53,131]$.) Nor will merely adding more overlapping response channels automatically improve the reconstruction in this technique since numerical instabilities and error amplification thereby tend to increase [82]. More advanced, regulated methods were cited in Sec. IIC. (3) Yet, even if the histogram reconstruction here is unacceptable in a pointwise sense, it may still prove useful as a constraint for more sophisticated regulated reconstructions [132]. (4) When different unfold algorithms (applied to the same data and response functions) yield differing results, the disagreement is often rooted in the embedded a priori rules for treating null spectra [Eq. (6)]. Since the response functions are per se insensitive to null spectra (Sec. II C), such conflict may be irresolvable without close evaluations of the algorithms. (5) As noted in Sec. III G, the analysis thus far has only cursorily estimated the magnifying effects of uncertainties in the unfold algorithm used here. Specifically, the measure cond $(\mathbb{R})$ sets only a broad-based upper bound to error propagation: e.g., $\left(\|\Delta \mathbf{S}\|\|\mathbf{S}\|^{-1}\right) \times$ $\left(\|\Delta \mathbf{D}\|\|\mathbf{D}\|^{-1}\right)^{-1} \leq \operatorname{cond}(\mathbb{R}) \sim 11$, for errors $\Delta \mathbf{D}$ in $\mathbf{D}$. This estimate is adequate for assessing unfold distortions with noise-free data (Sec. IV). But, in experiments, one desires error estimates for all the unfold components $S_{j}$ and the flux $\mathcal{F}_{\text {unfold }}$. This issue is examined in part 2 (Sec. II) for data noise and calibrational uncertainties. (6) Another yetto-be-resolved issue in this article is this: only selected spectral functions (e.g., those satisfying the assumptions of Table I) have been used to test algorithmic distortion. It is not clear how the algorithm in general responds to spectra that violate these assumptions. Nor, for that matter, do the present simulations quantitatively explain and predict the noted properties of the unfold algorithm, especially negative behavior. Part 2, Sec. III, resolves these and other issues for arbitrary spectral functions without explicitly constructing simulated data. A condition for applying this generalized approach to other unfold procedures is noted.

\section{ACKNOWLEDGMENTS}

It is a pleasure to note the contributions of Harry Kornblum (Lawrence Livermore Laboratories), Tom Tunnel (NSTech), Ray Dukart, Jim Bailey, and especially Ray Leeper. We are also grateful to our reviewers for their comments and for additional references. This work was performed by Sandia National Laboratories. Sandia is a multiprogram laboratory operated by Sandia Corporation, a Lockheed Martin Company, for the United States Department of Energy under Contract No. DE-AC0494AL85000.

\section{APPENDIX A: DATA CONVERSIONS AND THE TOTAL EMITTED POWER}

For convenience, the recorded voltage signals $V_{i}(t)$ are converted into the channel data $D_{i}(t)$ of Eq. (1). For example, the $Z$ diagnostic presents a solid angle, $\Omega_{i}[\mathrm{sr}]=$ $A_{\text {detector }, i} r^{-2}$, to the source, where $A_{\text {detector, } i}\left[\mathrm{~cm}^{2}\right]$ is the defining area of the $i$ th filtered-XRD channel and $r$ is the distance to the source; $\Omega_{i}$ is offset from the normal to the $z$-pinch axis by angle $\vartheta$. If the area of the source $A_{\text {source }}$ $\left[\mathrm{cm}^{2}\right]$ is known, appropriate units of $S(E)$ are $\mathrm{W} \mathrm{sr}{ }^{-1} \mathrm{~cm}^{-2} \mathrm{eV}^{-1}$. But, if the source area is unknown (e.g., for bare $z$ pinches), $A_{\text {source }}$ is absorbed into the incident spectrum and the units are $\mathrm{W} \mathrm{sr}^{-1} \mathrm{eV}^{-1}$. The responses $R_{i}(E)$ represent the spectrally dependent current produced per unit incident $\mathrm{x}$-ray power [typically, $\left.\sim \mu \mathrm{A} \mathrm{W}^{-1}\right]$ on an assembled filtered-detector channel. During irradiation, the XRD currents enter a cable system of impedance $z_{0}$ [ohms] and are recorded (after cable compensation and temporal alignment) as voltage signals $V_{i}(t)$. For bare $z$ pinches,

$$
\frac{V_{i}(t)}{z_{0}}=\alpha \int_{0}^{E_{\mathrm{MAX}}} S(E, t)\left[\Omega_{i} \cos \vartheta\right] R_{i}(E) d E,
$$


where $\alpha=10^{-6} \mathrm{~A} / \mu \mathrm{A}$ (units' conversion). The cosine term references the diagnostic to normal viewing of the $z$ pinch $\left(\vartheta=11.8^{\circ}\right)$. If one divides out the known experimental constants in Eq. (A1), one obtains the channel data for Eq. (1):

$$
D_{i}(t)=\int_{0}^{E_{\mathrm{MAX}}} R_{i}(E) S(E, t) d E=\kappa V_{i}(t),
$$

where

$$
\kappa_{i}=\frac{1}{\alpha z_{0} \Omega_{i} \cos \vartheta}\left[\mathrm{sr}^{-1} \mathrm{ohm}^{-1}\right] .
$$

Typically, $z_{0}=50 \mathrm{ohms}, A_{\text {detector }, i} \approx 0.1 \mathrm{~cm}^{2}, r=2390 \mathrm{~cm}$, and $\Omega_{i} \approx 1.73 \times 10^{-8} \mathrm{sr}$; hence, $\kappa_{i} \approx 1.2 \times 10^{12} \mathrm{sr}^{-1}$ $\mathrm{ohm}^{-1}$ and is consistent with $S$ in units of $\mathrm{W} \mathrm{sr}^{-1} \mathrm{eV}^{-1}$. For $z$ pinches enclosed in a hohlraum and viewed through a source aperture, $A_{\text {source }}$ is not implicit in $S$ and yields the data-conversion factor, $\kappa_{i}^{\prime}=\kappa_{i} / A_{\text {source }}$. In this case, $\kappa_{i}^{\prime}$ and $S$ have units of $\mathrm{sr}^{-1} \mathrm{ohm}^{-1} \mathrm{~cm}^{-2}$ and $\mathrm{W} \mathrm{sr}^{-1} \mathrm{~cm}^{-2} \mathrm{eV}^{-1}$, respectively [cf. $S_{b b}(E, T)$ in Eq. (7)].

For a bare $z$ pinch, the total power $P_{\text {tot }}[\mathrm{W}]$ emitted into $4 \pi$ sr can be inferred from a measurement of the x-ray flux $\mathcal{F}_{\text {unfold }}\left[\mathrm{W} \mathrm{sr}{ }^{-1}\right]$ and a simple plasma model. If the pinch is taken as a spatially uniform Lambertian emitter, then integrating over all emission angles one obtains

$$
P_{\text {tot }}(t)=\frac{\pi^{2}}{f_{\ell}} \mathcal{F}_{\text {unfold }}(t)
$$

where $f_{\ell}$ is the fraction of the $z$ pinch observed by the $Z$ diagnostic. (A slotted aperture, partially masking the vertical field of view of the $Z$ diagnostic, is often fielded near the pinch.) The constant $\pi^{2}$ derives from the assumption of Lambertian emission, which would be replaced by $4 \pi$ for an isotropic source. A $\cos \vartheta$ term does not appear in Eq. (A4) because it has already been included in $\mathcal{F}_{\text {unfold }}$ through Eq. (A3).

\section{APPENDIX B: CALIBRATION MODELS FOR THE RESPONSE FUNCTIONS $R_{i}(E)$}

Electron photoemission studies between $100 \mathrm{eV}$ and $10 \mathrm{keV}$ by Henke [133] show that in first order $\chi_{i}(E)$ in Eq. (2) is proportional to the photoelectric mass absorption coefficient of atoms close to the photocathode surface. Hence, in calibrations [56], the measured electrical current produced by the XRD per unit incident $\mathrm{X}$-ray power is fit to

$$
\chi_{i}(E)=\sum_{l} A_{i l} \mu_{i l}(E)
$$

where the absorption coefficients [64] $\mu_{i l}(E)\left[\mathrm{cm}^{2} \mathrm{~g}^{-1}\right]$ are known, and the constants $A_{i l}\left[\mathrm{~A} \mathrm{~W}^{-1} \mathrm{~g} \mathrm{~cm}^{-2}\right.$ ] result from a least-squares (LS) fit procedure. The sum over $l$ in Eq. (B1) is taken over the various chemical species on the photocathode: $\mathrm{C}$ dominates but $\mathrm{H}, \mathrm{O}$, and $\mathrm{Si}$ must be included as surface contaminants. Uncertainties of $\sim 10 \%-15 \%$ are typically ascribed to the $A_{i l}$ 's [56], which are correlated statistically by the fitting process. It is assumed that the photoelectric properties of the substrate are spatially uniform. Compton interactions modify this model for x-ray energies in excess of $\sim 30 \mathrm{keV}$.

The attenuations $\Theta_{i}(E)$ in Eq. (2) have been variously characterized experimentally [56]. At soft $\mathrm{x}$-ray energies the spectral transmission model in the $i$ th filter is taken as

$$
\ln \left[\Theta_{i}(E)\right]=-\sum_{m} \tau_{i m} \mu_{i m}(E)
$$

where $\mu_{i m}(E)$ is the mass photoelectric coefficient [64] of the $m$ th chemical species $(\mathrm{H}, \mathrm{Be}, \mathrm{C}, \mathrm{Zn}, \ldots)$ in the filter(s) [56], and $\tau_{i m}$ is the corresponding areal thickness $\left(\mathrm{g} \mathrm{cm}^{-2}\right)$. The uncertainty in $\tau_{i m}$ is estimated to be $\sim 5 \%$ [56]. Again, spatial uniformity of the filters is assumed, and Compton corrections may be necessary for $\mathrm{x}$ rays in excess of a $30 \mathrm{keV}$, depending on the filter material. The form and spectral range of the overall channel responses can be adapted to different $\mathrm{x}$-ray sources by adjusting the array components. (A separate unfold analysis is then performed for each configuration and calibration.)

Compatibility with Eq. (1) requires certain implicit, x-ray flux restrictions [62] so that $d_{i}(t)$ during irradiation derives solely from $S(E, t)$. For example, (a) there is insufficient flux to cause rapid changes in the responses $R_{i}$; (b) x rays of various energies are detected independently; and (c) the detectors are not driven into saturation.

\section{APPENDIX C: DEFINING AN EFFECTIVE UNFOLD DOMAIN $[\Delta E]$}

This analysis begins by normalizing each spectrum $S_{b b}(E, T)$ by its integral in $\left[0, E_{\mathrm{MAX}}\right]$ so that nonzero multiplicative scalars are excluded. The result, $S_{b b}(E, T) /$ $\int_{0}^{E_{\mathrm{MAX}}} S_{b b}\left(E^{\prime}, T\right) d E^{\prime}$, for each $T$ is called the normalized shape $\underline{S}_{b b}(E, T)$ of $S_{b b}(E, T)$, for $E \leq E_{\mathrm{MAX}}$. The corresponding normalized data are thus simulated as $\underline{d}_{i}[T]=$ $\int_{0}^{E_{\mathrm{MAX}}} R_{i}(E) \underline{S}_{b b}(E, T) d E$ for each channel $i$.

One next considers the partial contribution, $\int_{E_{\mathrm{LO}}}^{E_{\mathrm{HI}}} R_{i}\left(E^{\prime}\right) \underline{S}_{b b}\left(E^{\prime}, T\right) d E^{\prime}$, to $\underline{d}_{i}[T]$ for parameters, $E_{\mathrm{LO}}$ and $E_{\mathrm{HI}} \quad\left(0 \leq E_{\mathrm{LO}} \leq E_{\mathrm{HI}} \leq E_{\mathrm{MAX}}\right)$. Our criterion in choosing them is that within $\left[E_{\mathrm{LO}}, E_{\mathrm{HI}}\right] \underline{S}_{b b}(E, T)$ must contribute at least a prescribed fraction $(1-\alpha)$ of the normalized channel data for all the specified spectral shapes and responses:

$$
\frac{\int_{E_{\mathrm{LO}}}^{E_{\mathrm{HI}}} R_{i}\left(E^{\prime}\right) \underline{S}_{b b}\left(E^{\prime}, T\right) d E^{\prime}}{\int_{0}^{E_{\mathrm{MAX}}} R_{i}\left(E^{\prime}\right) \underline{S}_{b b}\left(E^{\prime}, T\right) d E^{\prime}} \geq(1-\alpha),
$$

where $0 \leq \alpha<1$. We choose $\alpha=0.05$; hence, partial contributions from $\left[E_{\mathrm{LO}}, E_{\mathrm{HI}}\right]$ must account for at least $95 \%$ of the simulated data $[104,105]$. 


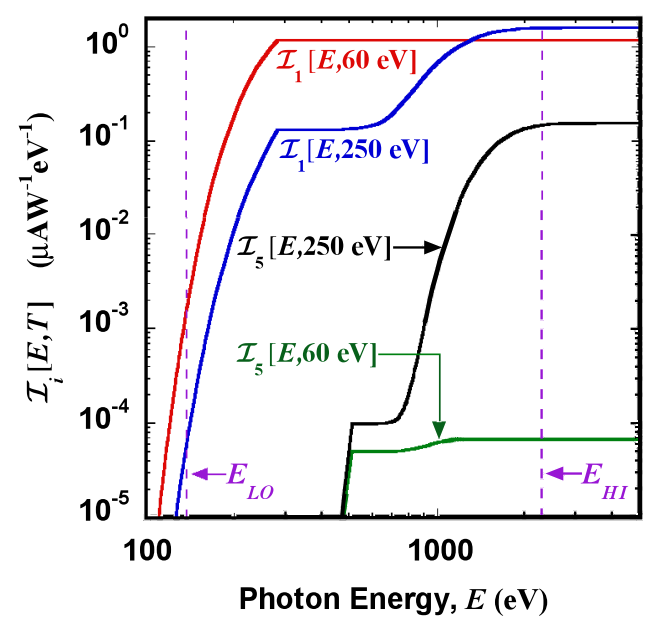

FIG. 13. Cumulative integrals of channel-wise spectrumresponse products over the interval $\left[0, E_{\mathrm{MAX}}\right]$ (cf. Fig. 5): $I_{i}[E, T]=\int_{0}^{E} R_{i}\left(E^{\prime}\right) \underline{S}_{b b}\left(E^{\prime}, T\right) d E^{\prime}$, where $\underline{S}_{b b}(E, T)$ is the normalized Planckian spectral shape for temperature $T$. The curves shown represent combinations of $i=1,5$ and $T=60,250 \mathrm{eV}$. (Similar results are obtained for intermediate channels and spectral temperatures but are not shown for clarity.) The righthand intercepts, $I_{i}\left[E_{\mathrm{MAX}}, T\right]$, are normalized data values, $\underline{d}_{i}[T]$, which differ from unity because only $\underline{S}_{b b}$ is normalized here, not $R_{i}(E) \underline{S}_{b b}(E)$. The vertical, dashed lines indicate the chosen unfold interval $[\Delta E]=\left[E_{\mathrm{LO}}, E_{\mathrm{HI}}\right]=[137 \mathrm{eV}, 2300 \mathrm{eV}]$.

Inequality (C1) can be rewritten more conveniently. Defining the channel- and shape-specific cumulative integrals, $I_{i}(E, T) \equiv \int_{0}^{E} R_{i}\left(E^{\prime}\right) \underline{S}_{b b}\left(E^{\prime}, T\right) d E^{\prime}$, one has

$$
\frac{I_{i}\left(E_{\mathrm{LO}}, T\right)}{\underline{d}_{i}[T]}+\frac{I_{i}\left(E_{\mathrm{MAX}}, T\right)-I_{i}\left(E_{\mathrm{HI}}, T\right)}{\underline{d}_{i}[T]} \leq \alpha,
$$

where $\underline{d}_{i}[T]=I_{i}\left(E_{\mathrm{MAX}}, T\right)$. It is now clear (a) that $E_{\mathrm{LO}}$ and $E_{\mathrm{HI}}$ can be determined independently (although not necessarily uniquely) and (b) that the shape $\underline{S}_{b b}(E, T)$ within $\left[E_{\mathrm{LO}}, E_{\mathrm{HI}}\right]$ does not appear explicitly in this determination, only its asymptotic behavior below and above $E_{\mathrm{LO}}$ and $E_{\mathrm{HI}}$, respectively.

Finally, one examines graphically where $I_{i}(E, T)$ complies with Eq. (C2) for all combinations of $\underline{S}_{b b}(E, T)$ and response functions $R_{i}(E)$. This process is illustrated in Fig. 13 for two extreme spectral shapes, $\underline{S}_{b b}(E, 60 \mathrm{eV})$ and $\underline{S}_{b b}(E, 250 \mathrm{eV})$, coupled to the least- and most-heavily filtered channel responses, $R_{1}(E)$ and $R_{5}(E)$, respectively. The corresponding simulated channel data $\underline{d}_{i}[T]$ over $\left[0, E_{\mathrm{MAX}}\right]$ are indicated at the right of the frame. The lower cutoff, $E_{\mathrm{LO}} \cong 137 \mathrm{eV}$, is suggested by Fig. 2, where $R_{1}(E \leq 137 \mathrm{eV}) \leq \max \left\{R_{1}\right\} / 100$. From Fig. 13, one finds that $I_{i}\left(E_{\mathrm{LO}} \equiv 137 \mathrm{eV}, T\right)$ amounts to less than $0.1 \%$ of the channel data across the combinations of channels and spectra considered. Similarly, the choice $E_{\mathrm{HI}} \equiv 2300 \mathrm{eV}$ yields $I_{i}\left(E_{\mathrm{MAX}}, T\right)-I_{i}\left(E_{\mathrm{HI}}, T\right)$ less than $5 \%$ of $\underline{d}_{i}[T]$. Hence, Eq. (C2) is satisfied. Thus, the lower unfold energy threshold is set by filtration in $R_{1}$, the upper by the highenergy tails of $R_{4}, R_{5}$, and $\underline{S}_{b b}(E, T=250 \mathrm{eV})$.

\section{APPENDIX D: INDEPENDENCE OF THE RESPONSE FUNCTIONS, THE GRAM MATRIX, SINGULAR-VALUE DECOMPOSITION, AND THE EXISTENCE OF SOLUTIONS TO EQS. (1) OR (5)}

The connection between linear independence of the responses and the condition of the Gram matrix $\mathbb{G}$ [Eq. (10)] follows from properties of the $R_{i}(E)$ 's $[52,53,62,106,107,111,112]$. Linear independence of the responses means that all linear combinations of the $R_{i}(E)$ 's are nonzero [i.e., $\sum_{i=1}^{N} \lambda_{i} R_{i}(E) \neq 0$ ], unless all the scalars $\lambda_{i} \equiv 0$ and except for a finite number of points. Now, since the $R_{i}(E)$ 's are here defined to be non-negative and not identically zero for the intervals of interest, independence of the responses is equivalent to $\sum_{i=1}^{N} \lambda_{i} \int R_{j}(E) R_{i}(E) d E \equiv \sum_{i=1}^{N} \lambda_{i} G_{j i} \neq 0 \quad$ with the same condition on the $\lambda_{i}$ 's, where the roles of $j, i$ may be interchanged. But, this result means that rows (and columns) of $\mathbb{G}$ must likewise be linearly independent and, hence, that $\mathbb{G}$ must be nonsingular. The inverse of this argument is also true.

To test the singularity of $\mathbb{G}$, it is convenient to factor $\mathbb{G}$ by the method of singular-value decomposition (SVD) $[49,51,61,74,75,82,83,96,97]$. The result is $\mathbb{G}=\mathbb{U} \tilde{\mathbb{G}} \mathbb{V}^{T}$, where $\mathbb{U}$ and $\mathbb{V}^{T}$ are orthogonal matrices $[106,107]$ and $\tilde{\mathbb{G}}$ is diagonal with diagonal elements, $g_{1}, \ldots, g_{N}$. The $g_{i}$ 's are the positive square roots of the eigenvalues of the product matrix $\mathbb{G}^{\mathrm{T}} \mathbb{G}$ and are called the singular values of $\mathbb{G}$. Since $\operatorname{det}(\mathbb{U})$, $\operatorname{det}\left(\mathbb{V}^{\mathrm{T}}\right)= \pm 1$, it follows that $|\operatorname{det}(\mathbb{G})|=g_{1} g_{2} \cdots g_{N}[114]$. Hence, in an analytical context, $\mathbb{G}$ is nonsingular-and the responses $R_{i}(E)$ are strictly independent-if, and only if, no $g_{k} \equiv 0$ [106-110].

In a numerical context, however, one must consider that $\mathbb{G}$ may be "nearly" or "effectively" singular $[53,62,63]$. That is, a numerical value $\operatorname{det}(\mathbb{G}) \approx 0$ may be caused either by one or more $g_{k}$ 's smaller than round-off error or by a scalar $\lambda \approx 0$, common to all the elements of $\mathbb{G}$ (i.e., $G_{i j}=$ $\lambda G_{i j}^{\prime}$ ), giving $|\operatorname{det}(\mathbb{G})|=\lambda^{N} g_{1}^{\prime} g_{2}^{\prime} \cdots g_{N}^{\prime} \approx 0$ [106]. One proposed metric for this judgment is to consider any $g_{k} \leq$ $N g_{1} \varepsilon_{\text {precision }}$ as effectively zero, where $N$ is the dimension of $\mathbb{G}, \varepsilon_{\text {precision }}$ is the floating-point precision, and $g_{1}$ is the largest singular value of $\mathbb{G}[82,97]$. The issue of small common scalars can then be eliminated by taking ratios $\left(g_{k} / g_{1}\right)$ : hence, $\left(g_{k} / g_{1}\right) \leq N \varepsilon_{\text {precision }}$ implies singularity in $\mathbb{G}$. According to IEEE standards [97], $\varepsilon_{\text {precision }} \approx 6 \times 10^{-8}$ (i.e., one part in $2^{24}$ ) for single precision calculations; hence, by the above criterion $\mathbb{G}(N=5)$ should be considered nearly singular if any ratio $\left(g_{k} / g_{1}\right) \leqslant 3 \times 10^{-7}$. This criterion explains the method of looking for sharp drops in $\left(g_{k} / g_{1}\right)$ vs $k$ in Fig. 6 . The condition number, also mentioned as a singularity test of $\mathbb{G}$, is cond $(\mathbb{G})=g_{1} / g_{N}$, 
where $g_{N}$ is the smallest singular value; $\mathbb{G}$ is said to be ill conditioned if the condition number $\operatorname{cond}(\mathbb{G}) \geq$ $\left(N \varepsilon_{\text {precision }}\right)^{-1} \approx\left(3 \times 10^{-7}\right)^{-1}=3 \times 10^{6}$.

Using the above standard for single precision, one may estimate the accumulated round-off error [Eq. (5)] for simulating data $d_{i}$ from, say, $\sim 1000$ spectral- and response-function points as between $\sim \sqrt{4000} \times \varepsilon_{\text {precision }}$ and $\sim 4000 \times \varepsilon_{\text {precision }}$, or $\sim 4 \times 10^{-6}-2 \times 10^{-4}$ [74].

To show the connection between independence in $\left\{R_{i}(E)\right\}_{i=1}^{N}$ and solutions to Eq. (5), assume first that the $R_{i}$ 's are linearly independent, choose arbitrary $d_{i}$, and define $f^{\dagger}(E)=\sum_{i=1}^{N} \Lambda_{i} R_{i}(E)[62,63]$, where the $\Lambda_{i}$ 's are to be determined. Substitution of $f^{\dagger}$ into Eq. (5) yields the $N$ simultaneous equations, $\sum_{j=1}^{N} G_{i j} \Lambda_{j}=d_{i}$, which have a unique solution because $\mathbb{G}$ is nonsingular since the $R_{i}$ 's are independent. This demonstrates at least one solution to Eq. (5). Conversely, assume that the $R_{i}$ 's are dependent [e.g., $R_{k}(E)=\sum_{i=1}^{M} \beta_{k, i} R_{i}(E)$ for $M<k \leq N$ ] and choose data components that differ in the $M-N$ redundant terms, $\sum_{i=1}^{M} \beta_{k, i} \int R_{i}(E) f(E) d E$, produced by all spectral functions $f(E)$ with these responses. Clearly, no exact solution to Eq. (5) can now exist because no $f(E)$ can reproduce the chosen data.

\section{APPENDIX E: DERIVATION OF EQS. (14) AND (26)}

The derivations of Eqs. (14) and (26) are based on assumed general behavior of $S(E)$ and $R_{i}(E)$ in the $j$ th unfold bin, $\left[E_{j}, E_{j+1}\right]=\left[\Delta E_{j}\right]$. (The reader will recall that these bin boundaries were selected, in part, to isolate known discontinuities in the $R_{i}$ 's, cf. Table II.) We assume that $S(E)$ and $R_{i}(E)$ are sufficiently well behaved to be expandable in Taylor series in each bin: e.g.,

$$
\begin{aligned}
S(E) \cong & S\left(\bar{E}_{j}\right)+S^{\prime}\left(\bar{E}_{j}\right)\left(E-\bar{E}_{j}\right)+\frac{1}{2 !} S^{\prime \prime}\left(\bar{E}_{j}\right)\left(E-\bar{E}_{j}\right)^{2} \\
& +\frac{1}{3 !} S^{\prime \prime \prime}\left(\bar{E}_{j}\right)\left(E-\bar{E}_{j}\right)^{3}+\cdots
\end{aligned}
$$

and similarly for each $R_{i}(E)$. Here $\bar{E}_{j}$ denotes the midpoint of $\left[\Delta E_{j}\right], \Delta E_{j}$ is the bin width, and the sequence $S^{\prime}\left(\bar{E}_{j}\right), S^{\prime \prime}\left(\bar{E}_{j}\right), \ldots$ indicates increasing-order derivatives of $S(E)$ evaluated at $\bar{E}_{j}$. When Eq. (E1) is integrated over $\left[\Delta E_{j}\right]$, one obtains

$$
\begin{aligned}
\int_{E_{j}}^{E_{j+1}} S(E) d E \cong & S\left(\bar{E}_{j}\right) \Delta E_{j}+0+\frac{1}{3} S^{\prime \prime}\left(\bar{E}_{j}\right)\left(\frac{\Delta E_{j}}{2}\right)^{3} \\
& +0+\cdots,
\end{aligned}
$$

where the odd-order terms in Eq. (E1) integrate to zero, due to our choice of expansion point $\bar{E}_{j}$. If $S\left(\bar{E}_{j}\right) \neq 0, S\left(\bar{E}_{j}\right) \Delta E_{j}$ may be factored out of the series to give Eq. (14).

Similar assumptions and analysis apply to Eq. (26), which is valid with the additional assumption that $S(E)$ is negligible outside $[\Delta E]$. The $\mathrm{x}$-ray data generated in Eq. (1) can then be written as

$$
d_{i} \equiv \int_{[\Delta E]} R_{i}(E) S(E) d E=\sum_{j=1}^{N} \int_{E_{j}}^{E_{j+1}} R_{i}(E) S(E) d E .
$$

[The perturbation terms $\varepsilon_{i}$ in Eq. (1) have been ignored.] Focusing on the contribution of the $j$ th bin to $d_{i}$, one writes

$$
\int_{E_{j}}^{E_{j+1}} R_{i}(E) S(E) d E=R_{i j}\langle S\rangle_{j} \frac{\int_{E_{j}}^{E_{j+1}} R_{i}(E) S(E) d E}{R_{i j}\langle S\rangle_{j}},
$$

where $R_{i j}=\int R_{i}(E) d E$ is integrated over $\left[\Delta E_{j}\right],\langle S\rangle_{j}=$ $\left[\Delta E_{j}\right]^{-1} \int_{E_{j}}^{E_{j+1}} S(E) d E$ is the average of $S(E)$ in $\left[\Delta E_{j}\right]$, and the product $R_{i j}\langle S\rangle_{j}$ is assumed to be nonzero. Expanding $S(E), R_{i}(E)$, and $S(E) R_{i}(E)$ about the midpoint $\bar{E}_{j}$ in the $j$ th bin and then performing the integrals as above, one finds

$$
\begin{aligned}
& \frac{\int_{E_{j}}^{E_{j+1}} R_{i}(E) S(E) d E}{R_{i j}\langle S\rangle_{j}} \\
& =\frac{1+\frac{\left(\Delta E_{j}\right)^{2}}{24}\left\{\frac{R_{i}^{\prime \prime}}{R_{i}}+2 \frac{R_{i}^{\prime}}{R_{i}} \frac{S^{\prime}}{S}+\frac{S^{\prime \prime}}{S}+\cdots\right\}}{\left\{1+\frac{\left(\Delta E_{j}\right)^{2}}{24} \frac{R_{i}^{\prime \prime}}{R_{i}}+\cdots\right\}\left\{1+\frac{\left(\Delta E_{j}\right)^{2}}{24} \frac{S^{\prime \prime}}{S}+\cdots\right\}} .
\end{aligned}
$$

A further binomial expansion of the denominators produces Eq. (26). Instead of using $\langle S\rangle_{j}$, one could also have simply factored out $S\left(\bar{E}_{j}\right)$ in Eq. (E4) as above with a corresponding adjustment to the expansion in Eq. (26).

[1] F. C. Jahoda, E. M. Little, W. E. Quinn, G. A. Sawyer, and T. F. Stratton, Phys. Rev. 119, 843 (1960).

[2] J. W. Mather, in Methods of Experimental Physics: Plasma Physics, edited by R.H. Lovberg and H. R. Griem (Academic Press, New York, 1971), Vol. 9B, and references therein.

[3] J. F. Kephart, R. P. Godwin, and G. H. McCall, Appl. Phys. Lett. 25, 108 (1974).

[4] V. W. Slivinsky, H. N. Kornblum, and H. D. Shay, J. Appl. Phys. 46, 1973 (1975).

[5] D. Attwood, Soft $X$ rays and Extreme Ultraviolet Radiation (Cambridge University Press, Cambridge, UK, 1999).

[6] J. P. Apruzese, J. Davis, K. G. Whitney, J. W. Thornhill, P. C. Kepple, R. W. Clark, C. Deeney, C. A. Coverdale, and T. W. L. Sanford, Phys. Plasmas 9, 2411 (2002).

[7] Y. B. Zel'dovich and Y. P. Raizer, Physics of Shock Waves and High-Temperature Hydrodynamic Phenomena, edited by W. D. Hayes and R. F. Probstein (Dover Publications, Mineola, New York, 2002); originally published by Academic Press, New York, in two volumes: 1966 and 1967, respectively.

[8] A.C. Phillips, The Physics of Stars (Wiley, New York, 1994).

[9] J.E. Bailey, P. Arnault, T. Blenski, G. Dehonghe, O. Peyrusse, J. J. MacFarlane, R. C. Mancini, M. E. Cuneo, D.S. Nielsen, and G. A. Rochau, J. Quant. Spectrosc. Radiat. Transfer 81, 31 (2003), and references therein. 
[10] J. W. Daiber and H. M. Thompson, J. Appl. Phys. 41, 2043 (1970).

[11] R. L. Kauffman, L. J. Suter, C. B. Darrow, J. D. Kilkenny, H. N. Kornblum, D. S. Montgomery, D. W. Phillion, M. D. Rosen, A. R. Theissen, R. J. Wallace, and F. Ze, Phys. Rev. Lett. 73, 2320 (1994).

[12] L. J. Suter, R. L. Kauffman, C. B. Darow, A. A. Hauer, H. Kornblum, O. L. Landen, T. J. Orzechowski, D. W. Phillion, J.L. Porter, L. V. Powers, A. Richard, M.D. Rosen, A. R. Thiessen, and R. Wallace, Phys. Plasmas 3, 2057 (1996), and references therein.

[13] M. S. Derzon, G. A. Chandler, R. J. Dukart, D. J. Johnson, R. J. Leeper, M. K. Matzen, E. J. McGuire, T. A. Mehlhorn, A. R. Moats, R.E. Olson, and C.L. Ruiz, Phys. Rev. Lett. 76, 435 (1996).

[14] D. L. Fehl, G. A. Chandler, F. Biggs, R. J. Dukart, A. R. Moats, and R. J. Leeper, Rev. Sci. Instrum. 68, 843 (1997).

[15] R. B. Spielman, C. Deeney, G. A. Chandler, M. R. Douglas, D. L. Fehl, M. K. Matzen, D. H. McDaniel, T. J. Nash, J. L. Porter, T. W. L. Sanford, J.F. Seamen, W. A. Stygar, K.W. Struve, S.P. Breeze, J.S. McGurn, J. A. Torres, D. M. Zagar, T.L. Gilliland, D. O. Jobe, J.L. McKenney, R.C. Mock, M. Vargas, and T. Wagoner, Phys. Plasmas 5, 2105 (1998), and references therein.

[16] J. E. Bailey, G. A. Chandler, D. Cohen, M. E. Cuneo, M. E. Foord, R. F. Heeter, D. Jobe, P. W. Lake, J. J. MacFarlane, T. J. Nash, D. S. Nielson, R. Smelser, and J. Torres, Phys. Plasmas 9, 2186 (2002).

[17] W. A. Stygar, H. C. Ives, D. L. Fehl, M.E. Cuneo, M. G. Mazarakis, J. E. Bailey, G. R. Bennett, D. E. Bliss, G. A. Chandler, R. J. Leeper, M. K. Matzen, D. H. McDaniel, J. S. McGurn, J. L. McKenney, L. P. Mix, D. J. Muron, J. L. Porter, J. J. Ramirez, L.E. Ruggles, J. F. Seamen, W. W. Simpson, C. S. Speas, R. B. Spielman, K. W. Struve, J. A. Torres, R. A. Vesey, T. C. Wagoner, T. L. Gilliland, M.L. Horry, D. O. Jobe, S. E. Lazier, J. A. Mills, T. D. Mulville, J. H. Pyle, T. M. Romero, J. J. Seamen, and R. M. Smelzer, Phys. Rev. E 69, 046403 (2004), and references therein.

[18] R. B. Spielman et al., in the 4th International Conference on Z Pinches, Vancouver, BC, Canada, AIP Conf. Proc. No. 409, edited by N. R. Pereira, J. Davis, and P. Pulsifer (AIP, New York, 1997), pp. 101-118.

[19] N. G. Basov and O. N. Krokhin, Sov. Phys. JETP 19, 123 (1964).

[20] K. A. Brueckner and S. Jorna, Rev. Mod. Phys. 46, 325 (1974).

[21] J. Lindl, Phys. Plasmas 2, 3933 (1995).

[22] W. A. Stygar, R.E. Olson, R. B. Spielman, and R. J. Leeper, Phys. Rev. E 64, 026410 2001), and references therein.

[23] R. J. Leeper, T.E. Alberts, J. R. Asay, P. M. Baca, K. L. Baker, S.P. Breeze, G. A. Chandler, D. L. Cook, G.W. Cooper, C. Deeney, M.S. Derzon, M.R. Douglas, D. L. Fehl, T. Gilliland, D. E. Hebron, M. J. Hurst, D. O. Jobe, J. W. Kellogg, J. S. Lash, S. E. Lazier, M. K. Matzen, D. H. McDaniel, J. S. McGurn, T. A. Mehlhorn, A.R. Moats, R. C. Mock, D. J. Muron, T. J. Nash, R.E. Olson, J.L. Porter, J.P. Quintenz, P. V. Reyes, L.E. Ruggles, C.L. Ruiz, T.W.L. Sanford, F. A. Schmidlapp, J.F. Seamen, R. B. Spielman, M. A. Stark, K. W. Struve, W. A. Stygar, D. R. Tibbetts-Russell, J. A. Torres, M. Vargas, T.C.
Wagoner, C. Wakefield, J. H. Hammer, D. D. Ryutov, M. Tabak, S.C. Wilks, R. L. Bowers, K. D. McLenithan, and D. L. Peterson, Nucl. Fusion 39, 1283 (1999).

[24] M. K. Matzen, Phys. Plasmas 4, 1519 (1997).

[25] T. J. Nash, M. S. Derzon, G. A. Chandler, D. L. Fehl, R. J. Leeper, J. L. Porter, R. B. Spielman, C. L. Ruiz, G. Cooper, J. McGurn, M. Hurst, D. Jobe, J. Torres, J. Seaman, K. Struve, S. Lazier, T. Gilliland, L. A. Ruggles, W. A. Simpson, R. Adams, J. A. Seaman, D. Wenger, D. Nielsen, P. Riley, R. French, B. Stygar, T. Wagoner, T.W.L. Sanford, R. Mock, J. Asay, C. Hall, M. Knudson, J. Armijo, J. McKenney, R. Hawn, D. Schroen-Carey, D. Hebron, T. Cutler, S. Dropinski, C. Deeney, P.D. LePell, C. A. Coverdale, M. Douglas, M. Cuneo, D. Hanson, J. E. Bailey, P. Lake, A. Carlson, C. Wakefield, J. Mills, J. Slopek, and T. Dinwoodie, Rev. Sci. Instrum. 72, 1167 (2001).

[26] J. R. Asay, C. A. Hall, C. H. Konrad, W. M. Trott, G. A. Chandler, K. J. Fleming, K. G. Holland, L. C. Chhabildas, T. A. Mehlhorn, R. Vesey, T. G. Trucano, A. Hauer, R. Cauble, and M Foord, J. Impact Engng. 23, 27 (1999).

[27] R. J. Lawrence, J. R. Asay, C. A. Hall, and T. G. Trucano, Shock Compression of Condensed Matter-1999, edited by M. D. Furnish, L.C. Chhabildas, and R.S. Hixson (American Institute of Physics, New York, 2000) p. 1079.

[28] Depending on context, the word spectrum here denotes any of the following radiometric quantities: the spectral radiant power $\left[\mathrm{W} \mathrm{eV}^{-1}\right]$; the spectral radiant intensity $\left[\mathrm{W} \mathrm{sr}-1 \mathrm{eV}^{-1}\right]$; the spectral radiance $\left[\mathrm{W} \mathrm{cm}^{-2} \mathrm{sr}^{-1}\right.$ $\left.\mathrm{eV}^{-1}\right]$; or the spectral irradiance in $\left[\mathrm{W} \mathrm{cm}^{-2} \mathrm{eV}^{-1}\right]$. While these quantities are, of course, physically distinct, from the standpoint of unfolding they differ by scalars and may be similarly addressed as long as the measuring units are consistent. The same comment applies to the term flux which here represents the integral of a spectrum over some $\mathrm{x}$-ray energy interval; corresponding units are $\mathrm{W}, \mathrm{W} \mathrm{sr}^{-1}$, $\mathrm{W} \mathrm{cm}{ }^{-2} \mathrm{sr}^{-1}$, and $\mathrm{W} \mathrm{cm}^{-2}$. See Appendix $\mathrm{A}$ and $\mathrm{K}$. Griepenkerl, S. Bohrmann, and K. Horn, in Handbook of Physics, edited by W. Benenson, J. W. Harris, H. Stocker, and H. Lutz (Springer, New York, 2002), p. 396 ff.

[29] D. L. Fehl, W. A. Stygar, G. A. Chandler, M. E. Cuneo, and C. L. Ruiz, Rev. Sci. Instrum. 76, 103504 (2005).

[30] D. L. Fehl et al., Rev. Sci. Instrum. 70, 270 (1999). Two confusing misprints appear in this article: " $2 \mathrm{~mJ}$ " should be replaced by "2 MJ" in Secs. I and II.

[31] H. N. Kornblum and V. W. Slivinsky, Rev. Sci. Instrum. 49, 1204 (1978).

[32] D. L. Hanson, (private communication).

[33] R. B. Spielman et al., Rev. Sci. Instrum. 70, 651 (1999).

[34] H. C. Ives et al., Phys. Rev. ST Accel. Beams 9, 110401 (2006).

[35] J.E. Monahan, in Scintillation Spectroscopy of Gamma Radiation, edited by S. M. Shafroth (Gordon and Beach, New York, 1967), Vol. 1, p. $371 \mathrm{ff}$.

[36] J.A.R. Samson, Techniques of Vacuum Ultraviolet Spectroscopy (Wiley, New York, 1967).

[37] H.R. Griem, Principles of Plasma Spectroscopy (Cambridge University Press, New York, 1997).

[38] L. E. Ruggles, J. L. Porter, Jr., and R. Bartlett, Rev. Sci. Instrum. 68, 1063 (1997).

[39] L. E. Ruggles et al., Rev. Sci. Instrum. 72, 1218 (2001). 
[40] J. F. Cuderman and K. M. Glibert, Rev. Sci. Instrum. 46, 53 (1975).

[41] R. B. Spielman, Rev. Sci. Instrum. 63, 5056 (1992).

[42] K. L. Baker, J. L. Porter, L. E. Ruggles, D. L. Fehl, G. A. Chandler, M. Vargas, L.P. Mix, W.W. Simpson, C. Deeney, R.E. Chrien, and G.C. Idzorek, Rev. Sci. Instrum. 70, 2012 (1999).

[43] C. L. Wang, Rev. Sci. Instrum. 53, 582 (1982).

[44] D. J. Johnson, Rev. Sci. Instrum. 45, 191 (1974).

[45] G. C. Idzorek and R. J. Bartlett, Proc. SPIE Int. Soc. Opt. Eng. 3114, 349 (1997).

[46] H. N. Kornblum, R. L. Kauffman, and J. A. Smith, Rev. Sci. Instrum. 57, 2179 (1986).

[47] R.L. Kaufman et al., Rev. Sci. Instrum. 66, 678 (1995).

[48] J. L. Bourgade, B. Villette, J. L. Bocher, J. Y. Boutin, S. Chiche, N. Dague, D. Gontier, J. P. Jadaud, B. Savale, R. Wrobel, and R.E. Turner, Rev. Sci. Instrum. 72, 1173 (2001).

[49] I. J. D. Craig and J.C. Brown, Inverse Problems in Astronomy: A Guide to Inversion Strategies for Remotely Sensed Data (Adam Hilger Ltd., Boston, 1986).

[50] C. de Mol, in Inverse Problems in Scattering and Imaging, Proceedings of a NATO Advanced Research Workshop, Cape Cod, USA, 1991, edited by M. Bertero and E. R. Pike (Adam Hilger, New York, 1992).

[51] M. Bertero and P. Boccacci, Introduction to Inverse Problems in Imaging (Institute of Physics Publishing, Philadelphia, 1998).

[52] G. M. Wing, A Primer on Integral Equations of the First Kind: The Problem of Deconvolution and Unfolding (SIAM, Philadelphia, 1991).

[53] S. Twomey, Introduction to the Mathematics of Inversion in Remote Sensing and Indirect Measurements (Elsevier, New York, 1977), reprinted with corrections by Dover Press, Mineola, NY, 1996.

[54] R. Kress, Linear Integral Equations (Springer-Verlag, New York, 1999), 2nd ed.

[55] D. L. Fehl, F. Biggs, G. A. Chandler, and W. A. Stygar, Rev. Sci. Instrum. 71, 3072 (2000).

[56] G. A. Chandler, C. Deeney, M. Cuneo, D. L. Fehl, J. S. McGurn, R. B. Spielman, J. A. Torres, J. L. McKinney, J. Mills, and K. W. Struve, Rev. Sci. Instrum. 70, 561 (1999).

[57] D. L. Fehl and F. Biggs, Rev. Sci. Instrum. 68, 890 (1997).

[58] G. C. Idzorek, R.E. Chrien, D. L. Peterson, R. G. Watt, G. A. Chandler, D. L. Fehl, and T. W. L. Sanford, Digest of Technical Papers, Pulsed Power Plasma Science Conference PPPS-2001 (IEEE, New York, 2002), Vol. 1, p. 777.

[59] E. M. Campbell, Laser Part. Beams 9, 209 (1991).

[60] E. M. Campbell, J. T. Hunt, E. S. Bliss, D. R. Speck, and R. P. Drake, Rev. Sci. Instrum. 57, 2101 (1986).

[61] M. Bertero, C. De Mol, and E. R. Pike, Inverse Probl. 4, 573 (1988).

[62] M. Bertero, C. De Mol, and E. R. Pike, Inverse Probl. 1, 301 (1985).

[63] M. Bertero, in Advances in Electronics and Electron Physics 75 edited by P.W. Hawkes (Academic Press, Harcourt Brace Jovanovich, Boston, 1989), pp. 2-120.

[64] F. Biggs and R. Lighthill, Sandia Report No. SAND870070.UC-34.
[65] The choice of an upper-bound x-ray energy $E_{\mathrm{MAX}}$ depends, of course, on the spectral sensitivity of the XRD's as well as the prominence of high-energy photons in the incident and scattered spectra. While $x$ rays of energy above $10 \mathrm{MeV}$ from tungsten $z$-pinch plasmas have been noted in high-gain detectors, other diagnostics indicate that $\mathrm{x}$ rays of energy greater than $\sim 10 \mathrm{keV}$ are relatively rare compared to $x$ rays of less than a few $\mathrm{keV}$. See Ref. [25], and R. B. Spielman, L. E. Ruggles, R. E. Pepping, S. P. Breeze, J.S. McGurn, and K.W. Struve, Rev. Sci. Instrum. 68, 782 (1997).

[66] G. A. Chandler et al., Rev. Sci. Instrum. 63, 4828 (1992).

[67] R. H. Day, P. Lee, E. B. Saleman, and D. J. Nagel, J. Appl. Phys. 52, 6965 (1981).

[68] R. B. Spielman and J. P. Anthes, AIP Conf. Proc. 75, 278 (1981).

[69] W. A. Stygar et al., Phys. Rev. E 72, 026404 (2005).

[70] W. A. Stygar et al., Phys. Rev. E 69, 046403 (2004).

[71] M. G. Haines, T. W. L. Sanford, and V. P. Smirnov, Plasma Phys. Controlled Fusion 47, B1 (2005).

[72] C. Deeney et al., Phys. Rev. E 56, 5945 (1997).

[73] P. M. Morse and H. Feshbach, Methods of Theoretical Physics, Pt. I (McGraw-Hill, New York, 1953).

[74] W. H. Press, S. A. Teukolsky, W. T. Vetterling, and B.P. Flannery, Numerical Recipes in FORTRAN: The Art of Scientific Computing (Cambridge University Press, New York, 1992), 2nd ed.

[75] R. Kress, Numerical Analysis (Springer, New York, 1998).

[76] R.P. Kanwal, Linear Integral Equations: Theory and Technique (Kirkhäuser, Boston, 1997), 2nd ed.

[77] F. G. Perey, Oak Ridge National Laboratory Report No. ORNL/TM-6062 ENDR-254, 1977.

[78] R. Gold, Argonne National Laboratory Report No. ANL6984, TID-4500, Argonne National Laboratory, Argonne, IL, 1964, 37th ed.

[79] S. G. Gorbics and N. R. Pereira, Rev. Sci. Instrum. 64, 1835 (1993).

[80] P. K. Lamm, in Proceedings of the First Conference in a Series on Inverse Problems in Engineering, Palm Coast, FL, 1993, edited by N. Zabaras, K. A. Woodbury, and M. Raynaud (The American Society of Mechanical Engineers, New York, 1993), p. 1.

[81] M. Bertero, in Inverse Problems in Scattering and Imaging, proceedings of a NATO Advanced Research Workshop held at Cape Cod, USA, 1991, edited by M. Bertero and E. R. Pike (Adam Hilger, Philadelphia, 1992).

[82] P.C. Hansen, Rank-Deficient and Discrete Ill-Posed Problems: Numerical Aspects of Linear Inversion (SIAM, Philadelphia, 1998).

[83] C. L. Lawson and R.J. Hanson, Solving Least Squares Problems (SIAM, Philadelphia, 1995).

[84] R. J. Hanson, SIAM J. Numer. Anal. 8, 616 (1971).

[85] C. W. Groetsch, The Theory of Tikhonov Regularization for Fredholm Equations of the First Kind (Pitman, Boston, 1984).

[86] C. W. Groetsch, Generalized Inverses of Linear Operators: Representation and Approximation (Marcel Dekker, New York, 1977).

[87] A. N. Tikhonov and V. Y. Arsenin, Solutions of Ill-Posed Problems (Wiley, New York, 1977). 
[88] P. A. Jansson, Deconvolution of Images and Spectra (Academic Press, San Diego, 1984), 2nd ed.

[89] A. Dermanis, A. Grün, and F. Sansò, Geomatic Methods for the Analysis of Data in the Earth Sciences (Springer, New York, 2000).

[90] R. Snieder and J. Trampert, in Geomatic Methods for the Analysis of Data in the Earth Sciences, edited by A. Dermanis, A. Grün, and F. Sansò (Springer, New York, 2000).

[91] R.L. Parker, Geophysical Inverse Theory (Princeton University Press, Princeton, NJ, 1994).

[92] T. P. Donaldson, Plasma Phys. 20, 1279 (1978).

[93] S. C. Sahasrabudhe and A. D. Kulkarni, J. Assoc. Comput. Mach. 24, 624 (1977).

[94] This fact is easily demonstrated in one dimension $(N=1)$ : let $d_{1}=\int R_{1}(x) f(x) d x$, where the integration interval of Eq. (5) is retained, $R_{1}(x) \equiv 1$, and $f(x)$ is chosen from the set of bounded, continuous functions. Then, finding the inverse $f(E)$ from a given $d_{1}$ fails because an infinite number of such functions $f(E)$ yield the same integral value. The possibility of no solution can be demonstrated in one dimension as well: e.g., there is no real $f(E)$ that maps to $d_{1}=$ $\left|\int R_{1}(E) f(E) d E\right|=-1$.

[95] Conversely, since the inversion of Eq. (1) as it stands is not unique, any two solutions, say $f_{1}$ and $f_{2}$-perhaps obtained by different unfolding methods-differ by a null function, which is invisible to the data. A nontrivial null function can be constructed from any (square-integrable) function $f(x)$ that is not a linear combination of the responses, $R_{i}(x)$. Let $y_{i}=\int_{a}^{b} R_{i}(x) f(x) d x$ [Eq. (5)] and define $f^{\dagger}(x)=\sum_{j=1}^{N} f_{j}^{\dagger} R_{j}(x)$; if the $R_{i}(x)$ 's are linearly independent, there is a unique solution for the coefficients $f_{j}^{\dagger}$. By substitution into Eq. (5), one finds that the difference function, $f(x)-\sum_{j=1}^{N} f_{j}^{\dagger} R_{j}(x)$, satisfies Eq. (6). See Refs. [51,61-63].

[96] G.E. Forsythe, M.A. Malcolm, and C.B. Moler, Computer Methods for Mathematical Computations (Prentice-Hall, Englewood Cliffs, NJ, 1977).

[97] D.S. Watkins, Fundamentals of Matrix Computations (Wiley, NY, 2002), 2nd ed.

[98] F. Reif, Fundamentals of Statistical and Thermal Physics (McGraw-Hill, New York, 1965).

[99] E. H. Bechner, J. Appl. Phys. 37, 4944 (1966).

[100] W. Priedhorsky and D. Lier, Rev. Sci. Instrum. 53, 1189 (1982).

[101] T. J. Murphy, R. A. Lerche, C. Bennett, and G. Howe, Rev. Sci. Instrum. 66, 930 (1995).

[102] Q. Xiao and G. Navratil, Rev. Sci. Instrum. 67, 3334 (1996).

[103] T. E. Lockard, G. C. Idzorek, T. E. Tierney, IV, and R. G. Watt, Rev. Sci. Instrum. 79, 10F322 (2008).

[104] S. L. Mayer, Data Analysis for Scientists and Engineers (Wiley, New York, 1975), p. 405 f.

[105] G. W. Snedecor and W. G. Cochran, Statistical Methods (Blackwell Publishing, Ames, Iowa, 1989), 8th ed.

[106] L. Råde and B. Westergren, Mathematics Handbook for Science and Engineering (Springer, New York, 2004), 5th ed.

[107] I. N. Bronshtein and K. A. Semendyayev, Handbook of Mathematics (Springer, New York, 1998), 3rd ed.
[108] K. Rektorys, in Survey of Applicable Mathematics edited by K. Rektorys (MIT Press, Cambridge, MA, 1969), p. 461.

[109] K. Rektorys, in Survey of Applicable Mathematics, edited by K. Rektorys (MIT Press, Cambridge, MA, 1969), pp. 691ff and 994ff.

[110] D.H. Griffel, Applied Functional Analysis (Dover Publications, Mineola, NY, 2002), originally published with revisions by Ellis Horwood Limited, Cichester, UK, 1985.

[111] R. Courant and D. Hilbert, Methods of Mathematical Physics (Interscience, New York, 1953), Vol. 1, 1st ed.

[112] N. I. Akhiezer and I. M. Glazman, Theory of Linear Operators in Hilbert Space (Dover Publications, Mineola, NY, 1993).

[113] A. Kirsch, An Introduction to the Mathematical Theory of Inverse Problems (Springer, New York, 1996).

[114] From Ref. [106], the determinant $\operatorname{det}(\mathbb{A B})=\operatorname{det}(\mathbb{A}) \times$ $\operatorname{det}(\mathbb{B}) ; \operatorname{det}(\mathbb{U})= \pm 1$ if $\mathbb{U}$ is a real, orthogonal

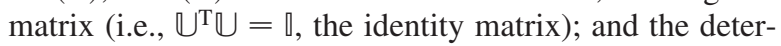
minant of a diagonal matrix is the product of the diagonal elements.

[115] G. E. Backus and J. F. Gilbert, Geophys. J. R. Astron. Soc. 13, 247 (1967).

[116] G. Backus and F. Gilbert, Geophys. J. R. Astron. Soc. 16, 169 (1968).

[117] G. Backus and F. Gilbert, Phil. Trans. R. Soc. A 266, 123 (1970).

[118] C. De Boor, A Practical Guide to Splines, \#27 in Applied Mathematical Science Series (Springer, NY, 1978).

[119] J. P. Knauer and N. C. Gindale, Rev. Sci. Instrum. 75, 3714 (2004).

[120] Jing Li, Xian-Bin Huang, Si-Qun Zhang, Li-Bing Yang, Wei-Ping Xie, and Yi-kang Pu, Rev. Sci. Instrum. 80, 063106 (2009).

[121] One rms measure of the relative closeness of a histogram, $\sum_{j=1}^{N} \beta_{j} B_{j}(E)$, to a given square-integrable spectral function $f(E)$ is $\min \left\|f-\sum_{j=1}^{N} \beta_{j} B_{j}\right\| /\|f\|$, where $\|*\|$ denotes the Euclidian norm over [0, $\left.E_{\mathrm{MAX}}\right]$. For the basis histogram functions $B_{j}(E)$ in Eq. (13), this minimization yields $\beta_{j}=\langle f\rangle_{j}$, and the definition of closeness reduces to $\left(1-\sum_{j}^{N}\langle f\rangle_{j}^{2} \Delta E_{j} / \int_{0}^{E_{\mathrm{MAX}}} f^{2} d E\right)^{1 / 2}$, which is 0 if $f(E)$ is a similar histogram and 1 if $f(E)$ is orthogonal to all the $B_{j}(E)$ 's.

[122] One can show that if the responses are linearly independent (as demonstrated by $\mathbb{G}$ ), and if the basis functions are linearly independent (as clearly chosen), and if no unfold basis function is orthogonal to all of the responses [i.e., $\left(R_{i}, B_{k}\right) \neq 0$ for any $k$ and for all channels $\left.i\right]$, then $\mathbb{R}$ will be nonsingular.

[123] M. J. Maron, Numerical Analysis (Macmillan, New York, 1987), 2nd ed.

[124] This conclusion results if $\mathbb{R}$ in Eqs. (18) and (19) is nonsingular and if all terms higher than the first in Eq. (26) are negligible. When these conditions hold, $\mathbb{R} \mathbf{S}_{\text {unfold }} \cong \mathbb{R} \mathbf{S}_{\mathrm{AVE}} ;$ hence, $\mathbb{R}\left(\mathbf{S}_{\text {unfold }}-\mathbf{S}_{\mathrm{AVE}}\right) \cong 0$, which implies that $\mathbf{S}_{\text {unfold }} \cong \mathbf{S}_{\mathrm{AVE}}$ (i.e., $S_{j} \cong\langle S\rangle_{j}$ ) for all unfold bins $j$. A more quantitative argument uses Pt. 2: Eqs. (33), (34), and (36), coupled to the Cauchy-Schwarz inequality, to find $\left|S_{j}-\langle S\rangle_{j}\right|=\left|\int_{0}^{E_{\mathrm{MAX}}} \rho_{U, j}(E) \Delta S_{\mathrm{B}}(E) d E\right| \leq$ $\left\|\rho_{U, j}\right\|\left\|\Delta S_{\mathrm{B}}\right\|$, where $\left\|\rho_{U, j}\right\|<\infty, \Delta S_{\mathrm{B}}(E)$ is the part of the source function $S(E)$ not approximated by the basis 
functions $\left\{B_{j}(E)\right\}_{j=1}^{N}$, and $\|S\|$ denotes the norm of function $S(E)$. If $S(E)$ is well approximated by the basis functions, $\left\|\Delta S_{\mathrm{B}}\right\| \rightarrow 0$ and $S_{j} \rightarrow\langle S\rangle_{j}$.

[125] D. L. Fehl, R. J. Leeper, and R.P. Kensek, Rev. Sci. Instrum. 63, 4786 (1992).

[126] Let spectrum $S(E)$ generate noise-free channel data $\mathbf{d}$ and $\tilde{\mathbf{d}}$ in two sets of responses $\left\{R_{i}(E)\right\}$ and $\left\{\tilde{R}_{i}(E)\right\}$, respectively; and assume that corresponding matrices, $\mathbb{R}$ and $\tilde{\mathbb{R}}$, constructed from Eq. (18), are nonsingular. Then one can expect unfolds, $\mathbf{S}_{\text {unfold }}$ and $\tilde{\mathbf{S}}_{\text {unfold }}$, to satisfy Eq. (19) for the pairs $\mathbb{R}$, d and $\tilde{\mathbb{R}}, \tilde{\mathbf{d}}$, respectively. Again using Pt. 2: Eqs. (33), (34), and (36), coupled to the Cauchy-Schwarz inequality, one obtains $\left|S_{j}-\langle S\rangle_{j}-\left(\tilde{S}_{j}-\langle S\rangle_{j}\right)\right|=\mid \int_{0}^{E_{\mathrm{MAX}}}$ $\left[\rho_{U, j}(E)-\tilde{\rho}_{U, j}(E)\right] \Delta S_{\mathrm{B}}(E) d E \mid \leq\left\|\rho_{U, j}-\tilde{\rho}_{U, j}\right\|\left\|\Delta S_{\mathrm{B}}\right\|$, where $\left\|\rho_{U, j}\right\|, \quad\left\|\rho_{U, j}\right\|<\infty$, and $\Delta S_{B}(E)$ is noted in Ref. [124] above. If $S(E)$ is well approximated, $\left\|\Delta S_{\mathrm{B}}\right\| \rightarrow 0$ and $S_{j} \rightarrow \tilde{S}_{j}$.

[127] L. Kissel, F. Biggs, and T. R. Marking, Sandia National Laboratories Report No. SAND82-0396, Sandia National Laboratories, Albuquerque, NM, 1991.
[128] Specifically, $\Omega(t)=\sqrt[4]{V_{1}(t) / \max V_{1}}$, where $V_{1}(t)$ is the channel-1 XRD voltage signal in $Z$-shot 179 , truncated at early and late times to avoid negative-valued baseline noise.

[129] G. C. Idzorek, G. A. Chandler, and D. L. Fehl, 25th IEEE Conference on Plasma Science (IEEE, New York, 1998), Conference Record Abstract 2P44, p. 163.

[130] H. F. Finn, Version 10/29/82, Lawrence Livermore National Laboratory Report No. UCID-19616, Livermore, CA, 1982.

[131] P.C. Hansen, Discrete Inverse Problems: Insight and Algorithms, Fundamentals of Algorithms (SIAM, Philadelphia, 2010).

[132] An example is the minimization of $\chi^{2}(t)=\sum_{i}\left[D_{i}(t)-\right.$ $\left.\int R_{i}(E) S_{\text {fit }}\left(E, t, c_{1}, \ldots, c_{M}\right) d E\right]^{2}+\lambda^{2} \sum_{j}\left[\int_{\left[\Delta E_{j}\right]} S_{\text {fit }}\left(E, t, c_{1}\right.\right.$, $\left.\left.\ldots, c_{M}\right) d E-\Delta E_{j} S_{j}\right]^{2}$, with respect to parameters $c_{1}, \ldots, c_{M}$. Here, $S_{\text {fit }}$ is a refined approximation to the incident spectrum, the $S_{j}$ 's are first-order histogram unfoldcoefficients, and $\lambda^{2}$ is a coupling constant (cf. Refs. [49,51,61-63]).

[133] B. L. Henke, J. A. Smith, and D. T. Attwood, J. Appl. Phys. 48, 1852 (1977). 